MATHEMATICS OF COMPUTATION

Volume 76, Number 260, October 2007, Pages 2189-2212

S 0025-5718(07)01986-2

Article electronically published on May 3, 2007

\title{
COMPUTING GRÖBNER FANS
}

\author{
KOMEI FUKUDA, ANDERS N. JENSEN, AND REKHA R. THOMAS
}

\begin{abstract}
This paper presents algorithms for computing the Gröbner fan of an arbitrary polynomial ideal. The computation involves enumeration of all reduced Gröbner bases of the ideal. Our algorithms are based on a uniform definition of the Gröbner fan that applies to both homogeneous and non-homogeneous ideals and a proof that this object is a polyhedral complex. We show that the cells of a Gröbner fan can easily be oriented acyclically and with a unique sink, allowing their enumeration by the memory-less reverse search procedure. The significance of this follows from the fact that Gröbner fans are not always normal fans of polyhedra, in which case reverse search applies automatically. Computational results using our implementation of these algorithms in the software package Gfan are included.
\end{abstract}

\section{INTRODUCTION}

The Gröbner fan of an ideal $I \subseteq k\left[x_{1}, \ldots, x_{n}\right]$ was defined by Mora and Robbiano in [19. It is a fan of polyhedral cones indexing initial ideals of $I$. The fulldimensional cones are in bijection with the distinct monomial initial ideals with respect to term orders or, equivalently, the reduced Gröbner bases of the ideal. In this paper we will describe algorithms for computing Gröbner fans of arbitrary polynomial ideals allowing us to study their structure in detail. Our algorithms are implemented in the software package Gfan [14].

The computation of the Gröbner fan of $I$ in terms of reduced Gröbner bases yields a universal Gröbner basis of $I$, a set of polynomials which is a Gröbner basis of $I$ with respect to every term order. The Gröbner fan also plays an important role in Gröbner basis conversion [6] and the emerging field of tropical mathematics as it contains the tropical variety of $I$ as a subfan [5. Many of the well-known theoretical applications of Gröbner bases rely on the existence of a Gröbner basis or initial ideal for an ideal with prescribed properties such as a particular complexity (a specified degree or squarefreeness) or homological properties (Cohen-Macaulay, Gorenstein, etc.). The (even partial) computation of the Gröbner fan makes such

Received by the editor September 23, 2005 and, in revised form, March 30, 2006.

2000 Mathematics Subject Classification. Primary 13P10, 52B20, 14Q99.

The research of the first author was supported by the Swiss National Science Foundation Project 200021-105202, "Polytopes, Matroids and Polynomial Systems".

The research of the second author was partially supported by the Faculty of Science, University of Aarhus, Danish Research Training Council (Forskeruddannelsesrådet, FUR), Institute for Operations Research ETH, the Swiss National Science Foundation Project 200021-105202, grants DMS 0222452 and DMS 0100141 of the U.S. National Science Foundation and the American Institute of Mathematics.

The research of the third author was supported by grant DMS 0401047 of the U.S. National Science Foundation.

(C)2007 American Mathematical Society Reverts to public domain 28 years from publication 
experimentations possible. No software package for the computation of Gröbner fans is available at present.

In the literature a distinction is often made between the case of $I$ being homogeneous, where the Gröbner fan is a complete fan in $\mathbb{R}^{n}$, and the case of $I$ being non-homogeneous, where the restricted Gröbner fan with support equal to $\mathbb{R}_{>0}^{n}$ is considered. In this paper we avoid this distinction by giving a new uniform definition of the Gröbner fan, and we prove that this actually defines a fan in the sense of a polyhedral complex - a proof that was left out in [19] but was proven for the special case of homogeneous ideals in [21. See Section 2].

In [21, Algorithms 3.2 and 3.6] and [19, Section 6] methods for computing the Gröbner fan of a polynomial ideal were given. In this paper we shall study 21, Algorithm 3.6] in detail. This algorithm traverses the maximal cones of the Gröbner fan. In the special case of toric ideals the traversal algorithm was already studied and implemented in [13. The traversal is graph-like - given a maximal cone we need to be able to find its facets, and we need to be able to walk through a facet to the neighboring maximal cone. Algorithms for performing these local computations are discussed in Section 4. These amount to solving linear programming problems and using the local basis change procedure due to [6]. We explain how to apply these methods to our case.

The Gröbner fan of a homogeneous ideal $I$ is known to be the normal fan of a polytope, the state polytope of $I$ ([21, Theorem 2.5]). In this homogeneous case traversal of the maximal cones in the Gröbner fan by walking through facets is equivalent to traversal of the edge graph of the state polytope. In [2] the memoryless reverse search procedure for traversing the edge graph of a polytope was given. This procedure easily applies to Gröbner fans of homogeneous ideals. However, the question is what happens if the ideal is not homogeneous. In [15] a non-regular Gröbner fan was presented - a fan that is not the normal fan of any polyhedron. In light of this example it is not clear that the reverse search technique applies to Gröbner fans in general. In Section 3 we prove that all Gröbner fans have what we shall call the reverse search property, allowing them to be traversed efficiently.

Gröbner fans are often computed for ideals that possess a great deal of symmetry. In Section 4.4 we describe how to take advantage of symmetry in the computations. The methods used here are similar to those in Rambau's software package TOPCOM [20] for traversing the secondary fan of a point configuration up to symmetry.

In Section 5 we discuss the complexity of our enumeration algorithm, and in Section 6 we present several examples of Gröbner fans computed using Gfan. This software package uses the GNU multi-precision library [12] for exact arithmetics and cddlib 9] for solving linear programming problems.

\section{The Gröbner fan of a POLYNomial ideal}

Let $R=k\left[x_{1}, \ldots, x_{n}\right]$ be the polynomial ring in $n$ variables over a field $k$ and let $I \subseteq R$ be an ideal. The Gröbner fan and the restricted Gröbner fan of $I$ are $n$-dimensional polyhedral fans defined in [19. We recall the definition of a fan in $\mathbb{R}^{n}$. A polyhedron in $\mathbb{R}^{n}$ is a set of the form $\left\{x \in \mathbb{R}^{n}: A x \leq b\right\}$ where $A$ is a matrix and $b$ is a vector. Bounded polyhedra are called polytopes. If $b=0$ the set is a polyhedral cone. The dimension of a polyhedron is the dimension of the smallest affine subspace containing it. A face of a polyhedron $P$ is either the empty set or a non-empty subset of $P$ which is the set of maximizers of a linear form over $P$. We 
use the following notation for the face maximizing a form $\omega \in \mathbb{R}^{n}$ :

$$
\text { face }_{\omega}(P)=\left\{p \in P:\langle\omega, p\rangle=\max _{q \in P}\langle\omega, q\rangle\right\} \text {. }
$$

A face of $P$ is called a facet if its dimension is one smaller than the dimension of $P$.

Definition 2.1. A collection $\mathcal{C}$ of polyhedra in $\mathbb{R}^{n}$ is a polyhedral complex if:

(1) all non-empty faces of a polyhedron $P \in \mathcal{C}$ are in $\mathcal{C}$, and

(2) the intersection of any two polyhedra $A, B \in \mathcal{C}$ is a face of $A$ and a face of $B$.

The support of $\mathcal{C}$ is the union of its members. A polyhedral complex is a fan if it only consists of cones. A fan is pure if all its maximal cones have the same dimension.

A simple way to construct a fan is to take the normal fan of a polyhedron.

Definition 2.2. Let $P \subseteq \mathbb{R}^{n}$ be a polyhedron. For a face $F$ of $P$ we define its normal cone

$$
N_{P}(F):=\overline{\left\{\omega \in \mathbb{R}^{n}: \text { face }_{\omega}(P)=F\right\}}
$$

with the closure being taken in the usual topology. The normal fan of $P$ is the fan consisting of the normal cones $N_{P}(F)$ as $F$ runs through all non-empty faces of $P$.

If the support of a fan is $\mathbb{R}^{n}$, the fan is said to be complete. It is clear that the normal fan of a polytope is complete. Not all fans arise as the normal fan of a polyhedron [11, page 25].

For $\alpha \in \mathbb{N}^{n}$ we use the notation $\mathbf{x}^{\alpha}:=x_{1}^{\alpha_{1}} \ldots x_{n}^{\alpha_{n}}$ for a monomial in $R$. By a term order on $R$ we mean a total ordering on all monomials in $R$ such that:

(1) for all $\alpha \in \mathbb{N}^{n} \backslash\{0\}: 1<\mathbf{x}^{\alpha}$ and

(2) for $\alpha, \beta, \gamma \in \mathbb{N}^{n}: \mathbf{x}^{\alpha}<\mathbf{x}^{\beta} \Rightarrow \mathbf{x}^{\alpha} \mathbf{x}^{\gamma}<\mathbf{x}^{\beta} \mathbf{x}^{\gamma}$.

By a term we mean a monomial together with its coefficient. Term orders are used for ordering terms, ignoring the coefficients. For a vector $\omega \in \mathbb{R}_{\geq 0}^{n}$ and a term order $\prec$ we define the new term order $\prec_{\omega}$ as follows:

$$
\mathbf{x}^{\alpha} \prec_{\omega} \mathbf{x}^{\beta} \Longleftrightarrow\langle\omega, \alpha\rangle\left\langle\langle\omega, \beta\rangle \vee\left(\langle\omega, \alpha\rangle=\langle\omega, \beta\rangle \wedge \mathbf{x}^{\alpha} \prec \mathbf{x}^{\beta}\right)\right. \text {. }
$$

Let $\prec$ be a term order. For a non-zero polynomial $f \in R$ we define its initial term, $\operatorname{in}_{\prec}(f)$, to be the unique maximal term of $f$ with respect to $\prec$. In the same way for $\omega \in \mathbb{R}^{n}$ we define the initial form, $\operatorname{in}_{\omega}(f)$, to be the sum of all terms of $f$ whose exponents maximize $\langle\omega, \cdot\rangle$. The polynomial $f$ is $\omega$-homogeneous $\operatorname{if}_{\omega}(f)=f$. The $\omega$-degree of a term $c \mathbf{x}^{\alpha}$ is $\langle\omega, \alpha\rangle$ and the $\omega$-degree of a non-zero polynomial $f$ is the common $\omega$-degree of the terms of $\operatorname{in}_{\omega}(f)$. The initial ideals of an ideal $I$ with respect to $\prec$ and $\omega$ are defined as

$$
\operatorname{in}_{\prec}(I)=\left\langle\operatorname{in}_{\prec}(f): f \in I \backslash\{0\}\right\rangle \text { and } \operatorname{in}_{\omega}(I)=\left\langle\operatorname{in}_{\omega}(f): f \in I\right\rangle .
$$

Note that $\operatorname{in}_{\prec}(I)$ is a monomial ideal while $\operatorname{in}_{\omega}(I)$ might not be. A monomial in $R \backslash \operatorname{in}_{\prec}(I)$ (with coefficient 1 ) is called a standard monomial of $\operatorname{in}_{\prec}(I)$.

Although initial ideals are defined with respect to not necessarily positive vectors, Gröbner bases are only defined with respect to true term orders:

Definition 2.3. Let $I \subseteq R$ be an ideal and $\prec$ a term order on $R$. A generating set $\mathcal{G}=\left\{g_{1}, \ldots, g_{m}\right\}$ for $I$ is called a Gröbner basis for $I$ with respect to $\prec$ if

$$
\operatorname{in}_{\prec}(I)=\left\langle\operatorname{in}_{\prec}\left(g_{1}\right), \ldots, \operatorname{in}_{\prec}\left(g_{m}\right)\right\rangle \text {. }
$$


The Gröbner basis $\mathcal{G}$ is minimal if $\left\{\operatorname{in}_{\prec}\left(g_{1}\right), \ldots, \operatorname{in}_{\prec}\left(g_{m}\right)\right\}$ generates $\operatorname{in}_{\prec}(I)$ minimally. A minimal Gröbner basis is reduced if the initial term of every $g \in \mathcal{G}$ has coefficient 1 and all other monomials in $g$ are standard monomials of in $\prec(I)$.

We use the term marked Gröbner basis for a Gröbner basis where the initial terms have been distinguished from the non-initial ones (they have been marked). For example, $\left\{\underline{x^{2}}+x y+y^{2}\right\}$ and $\left\{x^{2}+x y+y^{2}\right\}$ are marked Gröbner bases for the ideal $\left\langle x^{2}+x y+y^{2}\right\rangle$ while $\left\{x^{2}+x y+y^{2}\right\}$ is not since $x y$ is not the initial term of $x^{2}+x y+y^{2}$ with respect to any term order.

For a term order $\prec$ and an ideal $I$, Buchberger's algorithm guarantees the existence of a unique marked reduced Gröbner basis. We denote it by $\mathcal{G}_{\prec}(I)$. For two term orders $\prec$ and $\prec^{\prime}$, if $\operatorname{in}_{\prec}(I)=\operatorname{in}_{\prec^{\prime}}(I)$, then $\mathcal{G}_{\prec}(I)=\mathcal{G}_{\prec^{\prime}}(I)$. Conversely, given a marked Gröbner basis $\mathcal{G}_{\prec}(I)$, in $\prec(I)$ can be easily read off.

Given an ideal $I$, a natural equivalence relation on $\mathbb{R}^{n}$ is induced by taking initial ideals:

$$
u \sim v \Longleftrightarrow \operatorname{in}_{u}(I)=\operatorname{in}_{v}(I) .
$$

We introduce the following notation for the closures of the equivalence classes:

$$
\begin{gathered}
C_{\prec}(I)=\overline{\left\{u \in \mathbb{R}^{n}: \operatorname{in}_{u}(I)=\operatorname{in}_{\prec}(I)\right\}} \text { and } \\
C_{v}(I)=\overline{\left\{u \in \mathbb{R}^{n}: \operatorname{in}_{u}(I)=\operatorname{in}_{v}(I)\right\}} .
\end{gathered}
$$

Remark 2.4. It is well known that for a fixed ideal $I$ there are only finitely many sets $C_{\prec}(I)$ and they cover $\mathbb{R}_{>0}^{n}$; see [19. Secondly, every initial ideal in $\prec(I)$ is of the form $\operatorname{in}_{\omega}(I)$ for some $\omega \in \mathbb{R}_{>0}^{n}$; see [21, Proposition 1.11]. Consequently, every $C_{\prec}(I)$ is of the form $C_{\omega}(I)$.

A third observation is that the equivalence classes are not convex in general since we allow the vectors to be anywhere in $\mathbb{R}^{n}$ :

Example 2.5. Let $I=\langle x-1, y-1\rangle$. The ideal $I$ has five initial ideals: $\langle x-1, y-1\rangle$, $\langle x, y\rangle,\langle x, y-1\rangle,\langle x-1, y\rangle$ and $\langle 1\rangle$. In particular, for $u=(-1,3)$ and $v=(3,-1)$ we have $\operatorname{in}_{u}(I)=\operatorname{in}_{v}(I)=\langle 1\rangle$ but $\operatorname{in}_{\frac{1}{2}(u+v)}(I)=\langle x, y\rangle$.

Proposition 2.6. Let $\prec$ be a term order and $v \in C_{\prec}(I)$. For $u \in \mathbb{R}^{n}$,

$$
\operatorname{in}_{u}(I)=\operatorname{in}_{v}(I) \Longleftrightarrow \forall g \in \mathcal{G}_{\prec}(I), \operatorname{in}_{u}(g)=\operatorname{in}_{v}(g) .
$$

This proposition is a little more general than Proposition 2.3 in [21] as it allows the vectors $u$ and $v$ to have negative components. A proof is given in the next section. For fixed $\prec$ and $v$ as in Proposition 2.6, we get that $C_{v}(I)$, the closure of the equivalence class of $v$, is a polyhedral cone since each $g \in \mathcal{G}_{\prec}(I)$ introduces the equation $\operatorname{in}_{u}(g)=\operatorname{in}_{v}(g)$, which is equivalent to having $u$ satisfy a set of linear equations and strict linear inequalities; see Example 2.7. The closure is obtained by making the strict inequalities non-strict. Under the assumptions of Proposition 2.6 we may write this in the following way:

$$
u \in C_{v}(I) \Longleftrightarrow \forall g \in \mathcal{G}_{\prec}(I), \operatorname{in}_{v}\left(\operatorname{in}_{u}(g)\right)=\operatorname{in}_{v}(g) .
$$

As we saw in Example 2.5, not all equivalence classes are convex. However, for an arbitrary $v, C_{v}(I)$ is a convex polyhedral cone if it contains a strictly positive vector. In this case, there must exist a vector $p \in \mathbb{R}_{>0}^{n}$ in the interior of $C_{v}(I)$. Then $\operatorname{in}_{p}(I)=\operatorname{in}_{v}(I)$ and, by Lemma 2.15, $p \in C_{\prec p}(I)$ for any $\prec$. Hence the equivalence class of $v$ is of the form required in Proposition 2.6. 


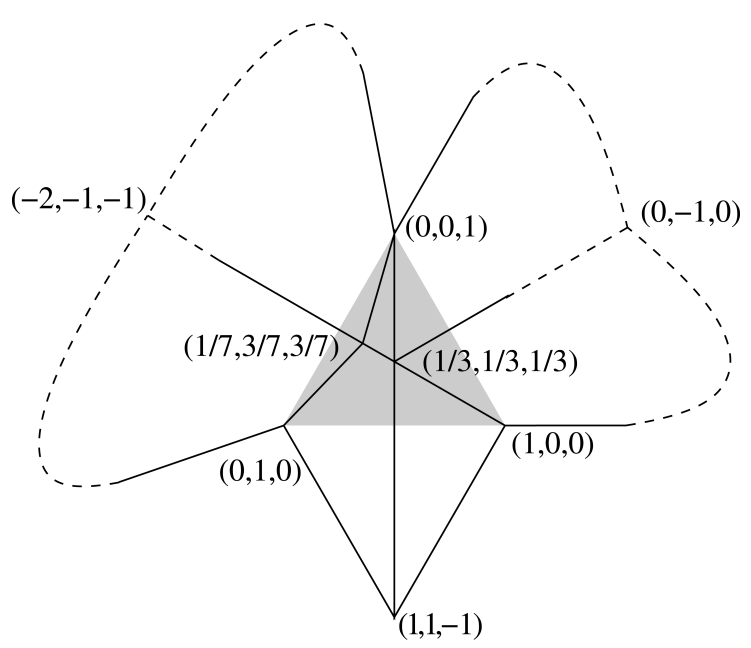

Figure 1. The Gröbner fan of the ideal in Example 2.7 has 7 three-dimensional, 14 two-dimensional and 8 one-dimensional cones. The intersections of the two-dimensional cones with the hyperplane $x+y+z=1$ are drawn as lines. The dotted part of the figure shows the combinatorial structure outside the hyperplane. The gray triangle indicates the positive orthant.

Example 2.7. Let $I=\left\langle x+y+z, x^{3} z+x+y^{2}\right\rangle \subseteq \mathbb{Q}[x, y, z]$ and let $\prec$ be the lexicographic term order with $x \prec y \prec z$. Then $\mathcal{G}_{\prec}(I)=\left\{\underline{y^{2}}+x-x^{3} y-x^{4}, \underline{z}+y+x\right\}$. If $v=(1,4,5)$, then $\operatorname{in}_{v}(I)=\operatorname{in}_{\prec}(I)=\left\langle y^{2}, z\right\rangle$ and $C_{v}(I)=C_{\prec}(I)$. By Proposition 2.6. $\operatorname{in}_{u}(I)=\operatorname{in}_{v}(I)$ if and only if the following two equations are satisfied:

$$
\begin{aligned}
\operatorname{in}_{u}(z+y+x) & =z\left(\Leftrightarrow u_{z}>\max \left\{u_{x}, u_{y}\right\}\right), \text { and } \\
\operatorname{in}_{u}\left(y^{2}+x-x^{3} y-x^{4}\right) & =y^{2}\left(\Leftrightarrow 2 u_{y}>\max \left\{u_{x}, 3 u_{x}+u_{y}, 4 u_{x}\right\}\right) .
\end{aligned}
$$

Introducing non-strict inequalities we obtain a description of $C_{\prec}(I)$. This cone is simplicial and has the cones $C_{(0,0,1)}(I), C_{(1,3,3)}(I)$ and $C_{(-2,-1,-1)}(I)$ as extreme rays and $C_{(1,3,4)}(I), C_{(-2,-1,0)}(I)$ and $C_{(-1,2,2)}(I)$ as facets. Since $(-2,-1,0)$ is in $C_{\prec}(I)$ a description of vectors $u$ in $C_{(-2,-1,0)}(I)$ is given by:

$$
\begin{gathered}
\operatorname{in}_{(-2,-1,0)}\left(\operatorname{in}_{u}(z+y+x)\right)=z\left(\Leftrightarrow u_{z} \geq \max \left\{u_{x}, u_{y}\right\}\right) \text {, and } \\
\operatorname{in}_{(-2,-1,0)}\left(\operatorname{in}_{u}\left(y^{2}+x-x^{3} y-x^{4}\right)\right)=y^{2}+x\left(\Leftrightarrow 2 u_{y}=u_{x} \geq \max \left\{3 u_{x}+u_{y}, 4 u_{x}\right\}\right) .
\end{gathered}
$$

Definition 2.8. The Gröbner fan of an ideal $I \subseteq R$ is the set of the closures of all equivalence classes intersecting the positive orthant together with their proper faces.

This is a variation of the definitions appearing in the literature. The advantage of this variant is that it gives well-defined and nice fans in the homogeneous and non-homogeneous case simultaneously. By nice we mean that all cones in this fan are closures of equivalence classes. It is not clear a priori that the Gröbner fan is a polyhedral complex. A proof is given in the next section (Theorem 2.19). The support of the Gröbner fan of $I$ is called the Gröbner region of $I$. Recall that the 

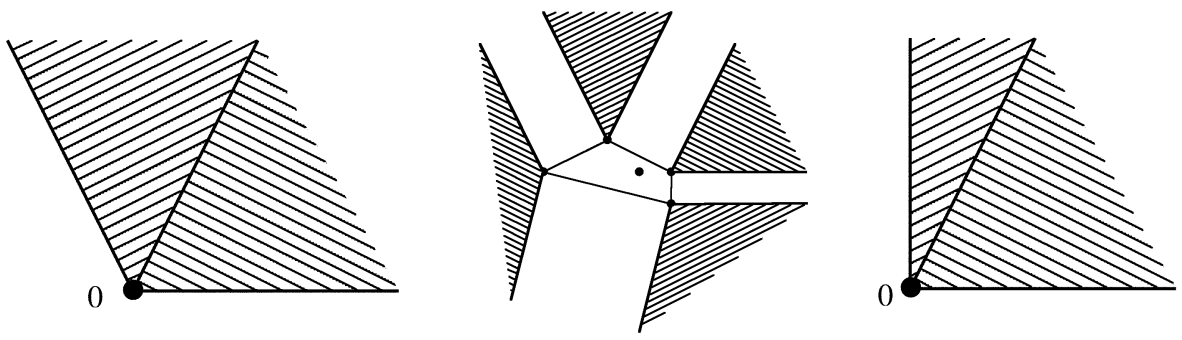

Figure 2. The Gröbner fan of the ideal in Example 2.9 is shown on the left. The restricted Gröbner fan is on the right. In the middle the Newton polytope of the generator is drawn with the shape of its normal fan indicated.

common refinement of two fans $\mathcal{F}_{1}$ and $\mathcal{F}_{2}$ in $\mathbb{R}^{n}$ is defined as

$$
\mathcal{F}_{1} \wedge \mathcal{F}_{2}=\left\{C_{1} \cap C_{2}\right\}_{\left(C_{1}, C_{2}\right) \in \mathcal{F}_{1} \times \mathcal{F}_{2}} .
$$

The common refinement of two fans is a fan. We define the restricted Gröbner fan of an ideal to be the common refinement of the Gröbner fan and the faces of the non-negative orthant. The support of the restricted Gröbner fan is $\mathbb{R}_{\geq 0}^{n}$. The Newton polytope of a polynomial is the convex hull of its exponent vectors.

Example 2.9. The Gröbner fan of the principal ideal $\left\langle x^{4}+x^{4} y-x^{3} y+x^{2} y^{2}+y\right\rangle$ consists of one 0 -dimensional cone, three 1-dimensional cones and two 2-dimensional cones; see Figure 2. The same is true for the restricted Gröbner fan. Notice, however, that in the restricted Gröbner fan one of the 1-dimensional cones and one of the 2-dimensional cones are not equivalence classes of the equivalence relation (2.1).

2.1. Proof: The Gröbner fan is a fan. In this section we prove that the Gröbner fan is a fan, i.e., that it is a polyhedral complex consisting of cones. Recall that the Gröbner fan, in general, is not complete and its support is larger than $\mathbb{R}_{>0}^{n}$. In 19] there is no proof that the Gröbner fan is a fan in the sense of a polyhedral complex. A proof that the Gröbner fan is a polyhedral complex under the assumption that the ideal is homogeneous is given in [21. We present a complete proof for the general case. Many of the results we need in the proof are generalizations of known results needed in the proof that the Gröbner fan of a homogeneous ideal is a polyhedral complex [21]. However, we do not rely on these references for the sake of being self-contained.

We fix the ideal $I \subseteq R$ in the following theorems. The most important step is the proof of Proposition 2.6 which tells us that the closure of an equivalence class is a polyhedral cone. Then we prove that the relative interior of any face in the Gröbner fan is an equivalence class (Proposition 2.16) and, finally, that the intersection of two cones in the fan is a face of both (Proposition 2.18).

To prove Proposition 2.6 we start by proving a similar statement for the equivalence classes arising from initial ideals with respect to term orders.

Lemma 2.10. Let $\prec$ be a term order. For $v \in \mathbb{R}^{n}$,

$$
\operatorname{in}_{v}(I)=\operatorname{in}_{\prec}(I) \Longleftrightarrow \forall g \in \mathcal{G}_{\prec}(I), \operatorname{in}_{v}(g)=\operatorname{in}_{\prec}(g) .
$$


Proof. $\Rightarrow$ : Let $g \in \mathcal{G}_{\prec}(I)$. Since $\mathcal{G}_{\prec}(I)$ is reduced, only one term from $g, \operatorname{in}_{\prec}(g)$, can be in $\operatorname{in}_{\prec}(I)=\operatorname{in}_{v}(I)$. The initial ideal $\operatorname{in}_{v}(I)$ is a monomial ideal, implying that all terms of an element in the ideal must be in the ideal too. Hence, the initial form $\operatorname{in}_{v}(g) \in \operatorname{in}_{v}(I)$ has to be equal to $\operatorname{in}_{\prec}(g)$.

$\Leftarrow$ : We must show that $\operatorname{in}_{v}(I)=\operatorname{in}_{\prec}(I)$, where $\operatorname{in}_{\prec}(I)=\left\langle\operatorname{in}_{\prec}(g)\right\rangle_{g \in \mathcal{G}_{\prec}(I)}$. The " $\supseteq$ " inclusion is clear since $\operatorname{in}_{\prec}(g)=\operatorname{in}_{v}(g) \in \operatorname{in}_{v}(I)$ for all $g \in \mathcal{G}_{\prec}(I)$.

To prove the " $\subseteq$ " inclusion, since $\operatorname{in}_{v}(I)=\left\langle\operatorname{in}_{v}(f), f \in I\right\rangle$, it suffices to show that $\operatorname{in}_{v}(f) \in \operatorname{in}_{\prec}(I)$ for all $f \in I$. Pick $f \in I$ and reduce it to zero using the division algorithm (e.g. [7, Chapter 2]) with $\mathcal{G}_{\prec}(I)$ and $\prec$. We may write

$$
f=m_{1} g_{i_{1}}+\cdots+m_{r} g_{i_{r}},
$$

where $m_{j}$ is a monomial and $g_{i_{j}}$ is an element from $\mathcal{G}_{\prec}(I)$. The division algorithm guarantees that $\operatorname{in}_{\prec}(f) \geq m_{j}$ in $_{\prec}\left(g_{i_{j}}\right)$ with respect to $\prec$ since monomials are substituted with monomials less than the original ones with respect to $\prec$ in the division process. Exactly the same thing is true for $v$-degrees since $v$ and $\prec$ agree on $\mathcal{G}_{\prec}(I)$. Thereby, any monomial on the right-hand side in (2.3) has $v$-degree less than or equal to the $v$-degree of the left-hand side. Consequently,

$$
\operatorname{in}_{v}(f)=\sum_{j \in J} m_{j} \operatorname{in}_{v}\left(g_{i_{j}}\right)
$$

with $j$ running through a subset such that $m_{j} \operatorname{in}_{v}\left(g_{i_{j}}\right)$ has the same $v$-degree as $\operatorname{in}_{v}(f)$. Since $\operatorname{in}_{v}(g) \in \operatorname{in}_{\prec}(I)$, the initial form $\operatorname{in}_{v}(f) \in \operatorname{in}_{\prec}(I)$.

By Lemma 2.10 the equivalence class of $\operatorname{in}_{\prec}(I)$ is open. Since $\operatorname{in}_{\prec}(I)$ is of the form $\operatorname{in}_{v}(I)$ for some $v$ (see Remark 2.4), the equivalence class of $\operatorname{in}_{\prec}(I)$ is also non-empty and hence full-dimensional. Thus we have proved that the equivalence class of a term order is a full-dimensional open polyhedral cone.

Corollary 2.11. Let $\prec$ be a term order and $v \in \mathbb{R}^{n}$. Then

$$
v \in C_{\prec}(I) \Leftrightarrow \forall g \in \mathcal{G}_{\prec}(I): \operatorname{in}_{\prec}\left(\operatorname{in}_{v}(g)\right)=\operatorname{in}_{\prec}(g) .
$$

Proof. Lemma 2.10 tells us that $v$ lies in the interior of $C_{\prec}(I)$ if and only if $\operatorname{in}_{v}(g)=$ in $_{\prec}(g)$ for all $g \in \mathcal{G}_{\prec}(I)$. Relaxing the resulting strict inequalities to non-strict inequalities we get a description of $C_{\prec}(I)$. This relaxation is exactly the one given by $\operatorname{in}_{\prec}\left(\operatorname{in}_{v}(g)\right)=\operatorname{in}_{\prec}(g)$ for all $g$ in $\mathcal{G}_{\prec}(I)$.

Lemma 2.12. A polynomial $f \in \operatorname{in}_{v}(I)$ can be written in the form $f=\sum_{i} \operatorname{in}_{v}\left(c_{i}\right)$, where $c_{i} \in I$ and all summands in the sum have different $v$-degrees.

Proof. The initial ideal $\operatorname{in}_{v}(I)$ is generated by $v$-homogeneous polynomials, implying that all $v$-homogeneous components of $f$ are in $\operatorname{in}_{v}(I)$. Let $h$ be a maximal $v$ homogeneous component of $f$. We need to show that $h$ is the initial form of an element in $I$ with respect to $v$. We may write $h$ as $\operatorname{in}_{v}\left(a_{1}\right)+\cdots+\operatorname{in}_{v}\left(a_{s}\right)$ for some polynomials $a_{1}, \ldots, a_{s}$ in $I$. Since $h$ is $v$-homogeneous we can rewrite $h$ as the sum $\sum_{j \in J} \operatorname{in}_{v}\left(a_{j}\right)$ of forms having the same $v$-degree as $h$. We pull out the initial form and get $h=\operatorname{in}_{v}\left(\sum_{j \in J} a_{j}\right)$.

Lemma 2.13. Let $\prec$ be a term order. If $v \in C_{\prec}(I)$, then $\operatorname{in}_{\prec}\left(\operatorname{in}_{v}(I)\right)=\operatorname{in}_{\prec}(I)$.

Proof. Let $g \in \mathcal{G}_{\prec}(I)$. Since $v \in C_{\prec}(I)$, by Corollary 2.11] $\operatorname{in}_{\prec}(g)=\operatorname{in}_{\prec}\left(\operatorname{in}_{v}(g)\right)$ and hence $\operatorname{in}_{\prec}(I)=\left\langle\operatorname{in}_{\prec}(g)\right\rangle_{g \in \mathcal{G}_{\prec}(I)} \subseteq \operatorname{in}_{\prec}\left(\operatorname{in}_{v}(I)\right)$. 
We now prove that $\operatorname{in}_{\prec}\left(\operatorname{in}_{v}(I)\right) \subseteq \operatorname{in}_{\prec}(I)$. Notice that $\operatorname{in}_{\prec}\left(\operatorname{in}_{v}(I)\right)$ is generated by initial terms of elements $f \in \operatorname{in}_{v}(I) \backslash\{0\}$ with respect to $\prec$. Suppose $f \in \operatorname{in}_{v}(I) \backslash\{0\}$. It suffices to show that $\operatorname{in}_{\prec}(f) \in \operatorname{in}_{\prec}(I)$. Using Lemma 2.12 we may write $f=$ $\sum_{i=1}^{s} \operatorname{in}_{v}\left(c_{i}\right)$, where $c_{1}, \ldots, c_{s} \in I$ and $\operatorname{in}_{v}\left(c_{1}\right), \ldots, \operatorname{in}_{v}\left(c_{s}\right)$ are $v$-homogeneous each with distinct degree, so that no cancellations occur. Consequently in $\prec(f)$ equals $\operatorname{in}_{\prec}\left(\operatorname{in}_{v}\left(c_{j}\right)\right)$ for some $j$. We wish to prove that $\operatorname{in}_{\prec}\left(\operatorname{in}_{v}\left(c_{j}\right)\right) \in \operatorname{in}_{\prec}(I)$. We use the division algorithm with $\mathcal{G}_{\prec}(I)$ and $\prec$ to rewrite $c_{j}$ as

$$
c_{j}=m_{1} g_{i_{1}}+\cdots+m_{r} g_{i_{r}},
$$

where $m_{1}, \ldots, m_{r}$ are monomials and $g_{i_{1}}, \ldots, g_{i_{r}}$ belong to $\mathcal{G}_{\prec}(I)$. Let $M$ be the $v$-degree of $c_{j}$. In the division algorithm we sequentially reduce $c_{j}$ to zero. In each step, the $v$-degree of $c_{j}$ will decrease or stay the same since we subtract the product of a monomial and an element from $\mathcal{G}_{\prec}(I)$, where the $v$-degree of the product already appeared in $c_{j}$ by Corollary 2.11. Equivalently, the product of the monomial and the element from $\mathcal{G}_{\prec}(I)$ are "added" to the right-hand side of the equation. We are done when $c_{j}=0$, or equivalently, the original $c_{j}$ is written as the above sum with every term having $v$-degree less than or equal to $M$. Consequently, we have

$$
\operatorname{in}_{v}\left(c_{j}\right)=\sum_{j^{\prime} \in J^{\prime}} \operatorname{in}_{v}\left(m_{j^{\prime}} g_{i_{j^{\prime}}}\right)
$$

for a suitable $J^{\prime}$. The division algorithm guarantees that the exponent vectors of in $_{\prec}\left(m_{1} g_{i_{1}}\right), \ldots$, in $_{\prec}\left(m_{r} g_{i_{r}}\right)$ are distinct. Since $v \in C_{\prec}(I)$, the monomials equal $\operatorname{in}_{\prec}\left(\operatorname{in}_{v}\left(m_{1} g_{i_{1}}\right)\right), \ldots, \operatorname{in}_{\prec}\left(\operatorname{in}_{v}\left(m_{r} g_{i_{r}}\right)\right)$. The maximal one of these with respect to $\prec$ cannot cancel in the sum. Hence $\operatorname{in}_{\prec}\left(\operatorname{in}_{v}\left(c_{j}\right)\right)=\operatorname{in}_{\prec}\left(m_{j^{\prime}} g_{i_{j^{\prime}}}\right)$ for some $j^{\prime}$, which implies that $\operatorname{in}_{\prec}\left(\operatorname{in}_{v}\left(c_{j}\right)\right) \in \operatorname{in}_{\prec}(I)$ as needed.

An easy corollary is a method for computing Gröbner bases for initial ideals.

Corollary 2.14. Let $\prec$ be a term order. If $v \in C_{\prec}(I)$, then

$$
\mathcal{G}_{\prec}\left(\operatorname{in}_{v}(I)\right)=\left\{\operatorname{in}_{v}(g)\right\}_{g \in \mathcal{G}_{\prec}(I)} .
$$

Proof. By Corollary 2.11, $\left\langle\operatorname{in}_{\prec}\left(\operatorname{in}_{v}(g)\right)\right\rangle_{g \in \mathcal{G}_{\prec}(I)}=\left\langle\operatorname{in}_{\prec}(g)\right\rangle_{g \in \mathcal{G}_{\prec}(I)}=\operatorname{in}_{\prec}(I)$. By Lemma 2.13, in $\prec(I)$ equals in $\prec\left(\operatorname{in}_{v}(I)\right)$. Thus in $\prec\left(\operatorname{in}_{v}(I)\right)=\left\langle\operatorname{in}_{\prec}\left(\operatorname{in}_{v}(g)\right)\right\rangle_{g \in \mathcal{G}_{\prec}(I)}$. This proves that $\left\{\operatorname{in}_{v}(g)\right\}_{g \in \mathcal{G}_{\prec}(I)}$ is a Gröbner basis of $\operatorname{in}_{v}(I)$ with respect to $\prec$. It is reduced since $\mathcal{G}_{\prec}(I)$ is minimal and reduced.

We are now able to give a proof for Proposition 2.6, which claimed that given $v \in C_{\prec}(I)$ and $u \in \mathbb{R}^{n}, \operatorname{in}_{u}(I)=\operatorname{in}_{v}(I) \Longleftrightarrow \forall g \in \mathcal{G}_{\prec}(I), \operatorname{in}_{u}(g)=\operatorname{in}_{v}(g)$.

Proof. $\Leftarrow$ : Since $\operatorname{in}_{u}(g)=\operatorname{in}_{v}(g)$ for all $g \in \mathcal{G}_{\prec}(I)$, we get that $\operatorname{in}_{\prec}\left(\operatorname{in}_{u}(g)\right)=$ $\operatorname{in}_{\prec}\left(\operatorname{in}_{v}(g)\right)$ for all $g \in \mathcal{G}_{\prec}(I)$. Since $v \in C_{\prec}(I)$, by Corollary [2.11, in in $_{\prec}(g)=$ $\operatorname{in}_{\prec}\left(\operatorname{in}_{v}(g)\right)$ for all $g \in \mathcal{G}_{\prec}(I)$ and hence in $\prec(g)=\operatorname{in}_{\prec}\left(\operatorname{in}_{u}(g)\right)$ for all $g \in \mathcal{G}_{\prec}(I)$ and $u \in C_{\prec}(I)$ by Corollary 2.11. The Gröbner basis $\mathcal{G}_{\prec}\left(\operatorname{in}_{u}(I)\right)$ is then $\left\{\operatorname{in}_{u}(g)\right\}_{g \in \mathcal{G}_{\prec}(I)}$ by Corollary [2.14. We get the same Gröbner basis for $\operatorname{in}_{v}(I)$. Hence, $\operatorname{in}_{u}(I)=$ $\operatorname{in}_{v}(I)$.

$\Rightarrow$ : Let $g \in \mathcal{G}_{\prec}(I)$. We need to show that $\operatorname{in}_{u}(g)=\operatorname{in}_{v}(g)$. Since the basis is reduced, only one term of $g$, namely $\operatorname{in}_{\prec}(g)$, is in in $_{\prec}(I)$. We start by proving that the term $\operatorname{in}_{\prec}(g)$ is a term in $\operatorname{in}_{v}(g)$ and a term in $\operatorname{in}_{u}(g)$. For $\operatorname{in}_{v}(g)$ we apply Corollary 2.11, which says $\operatorname{in}_{\prec}(g)=\operatorname{in}_{\prec}\left(\operatorname{in}_{v}(g)\right)$. For $\operatorname{in}_{u}(g)$ we apply Lemma 2.13 and get $\operatorname{in}_{\prec}\left(\operatorname{in}_{u}(g)\right) \in \operatorname{in}_{\prec}\left(\operatorname{in}_{u}(I)\right)=\operatorname{in}_{\prec}\left(\operatorname{in}_{v}(I)\right)=\operatorname{in}_{\prec}(I)$. Only one term of $g$ is in $\operatorname{in}_{\prec}(I)$, so in $\prec\left(\operatorname{in}_{u}(g)\right)=\operatorname{in}_{\prec}(g)$. If the difference $\operatorname{in}_{u}(g)-\operatorname{in}_{v}(g)$, belonging 
to $\operatorname{in}_{u}(I)=\operatorname{in}_{v}(I)$, is non-zero we immediately reach a contradiction since the difference contains no terms from in $\prec(I)=\operatorname{in}_{\prec}\left(\operatorname{in}_{v}(I)\right)$.

We have now proved that every equivalence class of a vector $v$ in a $C_{\prec}(I)$ is a relatively open convex polyhedral cone. By the argument following Proposition 2.6 in the previous section all sets in the Gröbner fan are in fact cones. We now argue that the relative interior of every cone in the Gröbner fan is an equivalence class.

Lemma 2.15. Let $\prec$ be a term order. If $v \in \mathbb{R}_{\geq 0}^{n}$, then $v \in C_{\prec v}(I)$.

Proof. This follows from Corollary 2.11 since $\operatorname{in}_{\prec_{v}}\left(\operatorname{in}_{v}(g)\right)=\operatorname{in}_{\prec_{v}}(g)$ for all $g \in$ $\mathcal{G}_{\prec v}(I)$.

Proposition 2.16. The relative interior of a cone in the Gröbner fan is an equivalence class (with respect to $u \sim u^{\prime} \Leftrightarrow \operatorname{in}_{u}(I)=\operatorname{in}_{u^{\prime}}(I)$ ).

Proof. By definition every cone in the fan is the face of the closure of an equivalence class for a positive vector $v \in \mathbb{R}_{>0}^{n}$. Let $\prec^{\prime}$ be an arbitrary term order and define $\prec$ as $\prec_{v}^{\prime}$. According to Lemma 2.15 the vector $v$ belongs to $C_{\prec}(I)$. Notice that by (2.2), $C_{v}(I) \subseteq C_{\prec}(I)$ since for all $u \in C_{v}(I)$ and $g \in \mathcal{G}_{\prec}(I)$, the condition $\operatorname{in}_{\prec}\left(\operatorname{in}_{u}(g)\right)=\operatorname{in}_{\prec}\left(\operatorname{in}_{v}\left(\operatorname{in}_{u}(g)\right)\right)=\operatorname{in}_{\prec}\left(\operatorname{in}_{v}(g)\right)=\operatorname{in}_{\prec}(g)$ of Corollary 2.11 is satisfied. By (2.2) the closed set $C_{v}(I)$ is cut out by some equations and non-strict inequalities. The relative interior of any face of $C_{v}(I)$ can be formed from this inequality system by changing a subset of the inequalities to strict inequalities and the remaining ones to equations. So let $u$ be a vector in the relative interior of some face of $C_{v}(I)$. The vector $u$ is in $C_{v}(I) \subseteq C_{\prec}(I)$. We may use Proposition 2.6 to conclude that a vector $u^{\prime} \in \mathbb{R}^{n}$ is equivalent to $u$ if and only if it satisfies the inequality system mentioned above - that is, if and only if it is in the relative interior of the face.

It remains to be shown that the intersection of two cones in the Gröbner fan is a face of both cones (Proposition 2.18). We need a few observations.

Corollary 2.17. Let $C$ be a cone in the Gröbner fan. If $v \in C$, then for $u \in \mathbb{R}^{n}$,

$$
\operatorname{in}_{u}(I)=\operatorname{in}_{v}(I) \Rightarrow u \in C .
$$

Proof. The vector $v$ is in the relative interior of some face of $C$. This face is also in the Gröbner fan. By Proposition 2.16 $u$ is in the relative interior of the same face and, consequently, also in $C$.

By Remark 2.4 there are only finitely many initial ideals given by term orders and, consequently, only finitely many reduced Gröbner bases of $I$. It follows that there can only be finitely many equivalence classes of the type described in Proposition 2.6 and Proposition 2.16.

Proposition 2.18. Let $C_{1}$ and $C_{2}$ be two cones in the Gröbner fan of I. Then the intersection $C_{1} \cap C_{2}$ is a face of $C_{1}$.

Proof. The intersection $C_{1} \cap C_{2}$ is a cone. By Corollary 2.17, $C_{1}$ and $C_{2}$ are unions of equivalence classes. Furthermore, if $v \in C_{1} \cap C_{2}$, then again by Corollary 2.17] the entire equivalence class of $v$ is both in $C_{1}$ and in $C_{2}$ and hence in $C_{1} \cap C_{2}$. Hence $C_{1} \cap C_{2}$ is a union of equivalence classes.

Let $u$ be a vector in such an equivalence class $E$ contained in $C_{1} \cap C_{2}$. Then $u$ is in the relative interior of one of the faces of $C_{1}$ which is a cone in the Gröbner fan. 
By Proposition 2.16 the set of vectors in the relative interior of this face is exactly $E$. Hence every such equivalence class is the relative interior of a face of $C_{1}$ and its closure is the face.

Look at the $\mathbb{R}$-span of each equivalence class contained in $C_{1} \cap C_{2}$. These spans must be different for every face of $C_{1}$. We claim that there can be only one maximaldimensional cone/span. If there were two cones, then their convex hull would be in $C_{1} \cap C_{2}$ and have dimension at least one higher and thus cannot be covered by the finitely many lower-dimensional equivalence classes - a contradiction.

Let $E$ be the maximal-dimensional equivalence class contained in $C_{1} \cap C_{2}$. We will argue that $\bar{E}=C_{1} \cap C_{2}$. The inclusion $\bar{E} \subseteq C_{1} \cap C_{2}$ is already clear since $C_{1} \cap C_{2}$ is closed. To prove the other inclusion suppose $\omega \in C_{1} \cap C_{2} \backslash \bar{E}$. Then $\operatorname{conv}(\bar{E}, \omega) \backslash \bar{E}$ is contained in $C_{1} \cap C_{2}$ and has dimension at least the dimension of $E$. This is a contradiction since $\operatorname{conv}(\bar{E}, \omega) \backslash \bar{E}$ cannot be covered by finitely many lower-dimensional equivalence classes. This completes the proof.

Theorem 2.19. The Gröbner fan is a polyhedral complex of cones and hence a fan.

Proof. We already argued using Proposition 2.6 and Lemma 2.15 that the Gröbner fan consists of polyhedral cones. The first condition for being a polyhedral complex is satisfied by definition. The second condition is Proposition 2.18,

\section{REVERSE SEARCH PROPERTY}

By the graph of a pure full-dimensional fan we mean the set of maximal cones with two cones being connected if they share a common facet. In this section we will prove that the reverse search technique [2] can be used for traversing the graph of a Gröbner fan. This follows from the main theorem, Theorem 3.6 which says that the graph of a Gröbner fan can be oriented easily without cycles and with a unique sink. In Definition 3.4 we define what we mean by this.

We start by explaining how a graph with this special kind of orientation can be traversed by reverse search. The idea is to define a spanning tree of the graph, which can be easily traversed. The following is a simple proposition, which we shall not prove.

Proposition 3.1. Let $G=(V, E)$ be an oriented graph without cycles and with a unique sink $s$. If for every vertex $v \in V \backslash\{s\}$ some outgoing search edge $e_{v}=(v, \cdot)$ is chosen, then the set of chosen edges is a spanning tree for $G$.

The spanning tree in Proposition 3.1 is referred to as the search tree. The proposition implies that the graph is connected.

Notice that we can find the sink by starting at any vertex and walking along a unique path of search edges until we get stuck, in which case we are at the sink. Consequently, the sink is the root of the oriented spanning tree. A corollary to the proposition is the reverse search algorithm for traversing $G$ :

Algorithm 3.2. Let $G=(V, E)$ be the oriented graph of Proposition 3.1 and suppose the choice of a search edge $e_{v}$ for each vertex $v \neq s$ has been made. Calling the following recursive procedure with $v=s$ will output all vertices in $G$. 


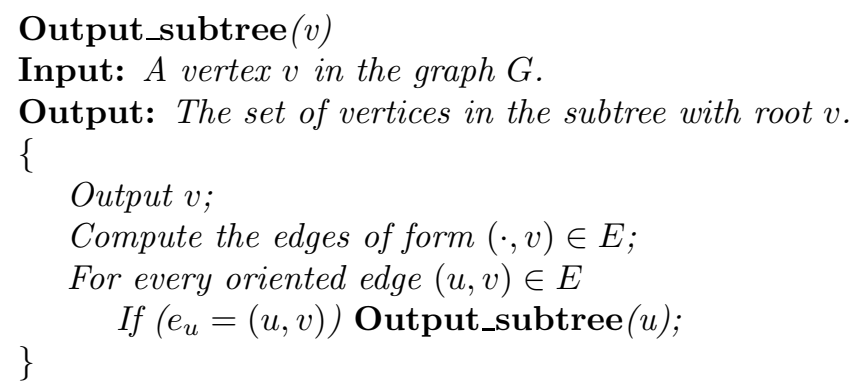

This algorithm does not have to store a set of "active" vertices as is usually needed in depth- and breadth-first traversals. It is even possible to formulate the algorithm completely without recursion avoiding the need for a recursion stack. In that sense the algorithm is memory-less.

We give an example of how the edge graph of a polytope or, equivalently, the graph of its normal fan can be oriented.

Example 3.3. Let $P \subset \mathbb{R}^{n}$ be a polytope whose vertices have positive integer coordinates and let $\prec$ be a term order on $R$. The following is an orientation of the edge graph of $P$ without cycles and with a unique sink: An edge $(p, q)$ is oriented from $p$ to $q$ if and only if $\mathbf{x}^{p} \prec \mathbf{x}^{q}$.

This defines an orientation of the graph of the normal fan of a polytope for any term order. We would like to mimic this orientation for any pure full-dimensional fan in $\mathbb{R}^{n}$. For simplicity we shall restrict ourselves to fans whose $(n-1)$-dimensional cones allow rational normals. In view of Propositions 2.6 and 2.16 this is no restriction for Gröbner fans.

Definition 3.4. A pure full-dimensional fan in $\mathbb{R}^{n}$ is said to have the reverse search property if for any term order $\prec$ the following is an acyclic orientation of its graph with a unique sink: If $\left(C_{1}, C_{2}\right)$ is an edge, then $C_{1}$ and $C_{2}$ are $n$-dimensional cones with a common facet $F$. Let $p, q \in \mathbb{N}^{n}$ such that $q-p \neq 0$ is a normal for $F$ with all points in $C_{1} \backslash F$ having negative inner product with $q-p$ and all points in $C_{2} \backslash F$ having positive inner product with $q-p$. We orient the edge in the direction from $C_{1}$ to $C_{2}$ if and only if $\mathbf{x}^{p} \prec \mathbf{x}^{q}$.

Note that the orientation of an edge in Definition 3.4 does not depend on the particular choice of $p$ and $q$. Note also that for normal fans of polytopes this orientation agrees with the orientation of the edge graphs of the polytopes in Example 3.3 . Not every fan has the reverse search property:

Example 3.5. Figure 3 shows a fan with support $\mathbb{R}_{\geq_{0}}^{3}$ intersected with the standard simplex. The intersection is the non-dotted part of the figure. For every shared 2-dimensional facet the orientation of its edge with respect to a term order of form $\prec_{(1,1,1)}$ is indicated by an arrow. The graph has a cycle. The reason is that the vector $(1,1,1)$ is in the interior of the cone over the dotted triangle and therefore induces the shown orientation with any tie-breaking.

Example 3.3 on the other hand shows that any normal fan of a polytope has the reverse search property. If $I$ is a homogeneous ideal, the Gröbner fan of $I$ is known to be the normal fan of the state polytope of $I$; see [21] for a proof. (We should mention that in [18] it was proven that this is only true if we use the state 


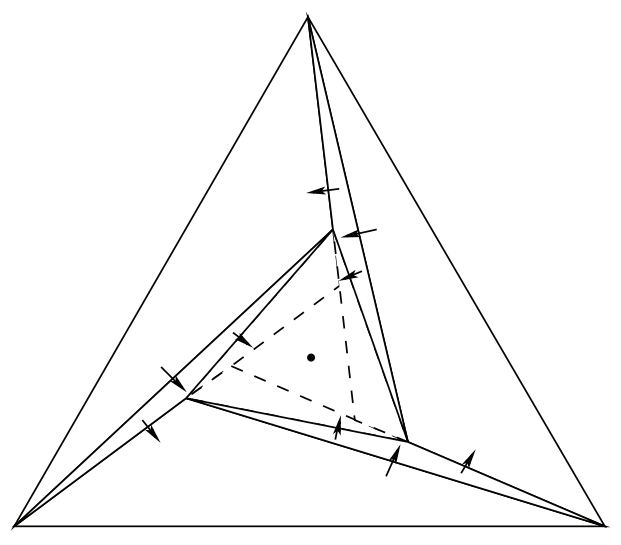

Figure 3. A fan not having the reverse search property; see Example 3.5

polytope definition in 21] and is not true with the original definition in 3.) As a consequence the Gröbner fan will have the reverse search property. The reverse search orientation of a fan with respect to any term order can be carried out on any fan covering $\mathbb{R}_{\geq 0}^{n}$ and being the normal fan of a polyhedron. Since the restricted Gröbner fan of any 0-dimensional or principal ideal satisfies these conditions it is clear that these fans have the reverse search property.

It is shown in [15] that this line of reasoning cannot be applied to Gröbner fans in general. In particular, an ideal is presented whose restricted Gröbner fan is not the normal fan of a polyhedron. For this reason we need a non-trivial argument to prove the following theorem:

Theorem 3.6. The Gröbner fan of any ideal $I \subseteq R$ has the reverse search property.

The proof is given in the next section. In Section 4 we will argue that all parts of Algorithm 3.2 (finding adjacent edges, finding adjacent vertices and finding search edges) can be implemented efficiently for Gröbner fans.

3.1. Proof: The Gröbner fan has the reverse search property. In this section we prove Theorem 3.6. We start by recalling how the polynomial ring can be graded by semigroups. This leads to a more general notion of homogeneous ideals.

Definition 3.7. By a grading on $R=k\left[x_{1}, \ldots, x_{n}\right]$ we mean a pair $(A, \mathcal{A})$ consisting of an abelian semigroup $A$ and a semigroup homomorphism:

$$
\mathcal{A}: \mathbb{N}^{n} \rightarrow A
$$

such that $\mathcal{A}^{-1}(a)$ is finite for all $a \in A$. The $\mathcal{A}$-degree of a term $c \mathbf{x}^{b}$ is $\mathcal{A}(b)$. A polynomial is $\mathcal{A}$-homogeneous if all its terms have the same $\mathcal{A}$-degree. An ideal is $\mathcal{A}$-homogeneous if it is generated by a set of $\mathcal{A}$-homogeneous polynomials.

For a grading $(A, \mathcal{A})$ on $R$ we get the direct sum of $k$-vector spaces

$$
R=\bigoplus_{a \in A} R_{a}
$$

where $R_{a}$ denotes the $k$-subspace of $R$ consisting of $\mathcal{A}$-homogeneous polynomials of degree $a$. Any reduced Gröbner basis of an $\mathcal{A}$-homogeneous ideal $I$ consists 
of $\mathcal{A}$-homogeneous polynomials. In particular, by generalizing the argument of Lemma 2.12 we get the direct sum

$$
I=\bigoplus_{a \in A} I_{a}
$$

where $I_{a}$ denotes the $k$-subspace of $I$ consisting of $\mathcal{A}$-homogeneous polynomials of degree $a$. The $\mathcal{A}$-homogeneous part $I_{a}$ is a $k$-subspace of $R_{a}$. We define the $\mathcal{A}$-graded Hilbert function:

$$
\begin{aligned}
H_{I, \mathcal{A}}: A & \rightarrow \mathbb{N} \\
a & \mapsto \operatorname{dim}_{k}\left(R_{a} / I_{a}\right) .
\end{aligned}
$$

Remark 3.8. For a monomial ideal $I$ the standard monomials of degree $a$ form a basis for $R_{a} / I_{a}$. Hence $H_{I, \mathcal{A}}(a)$ counts the number of standard monomials of degree $a$.

In general, as the following well-known proposition shows, the Hilbert function can be found by looking at a monomial initial ideal:

Proposition 3.9. Let $I$ be an $\mathcal{A}$-homogeneous ideal and $\prec$ a term order. Then

$$
H_{I, \mathcal{A}}=H_{\mathrm{in}_{\prec}(I), \mathcal{A}} .
$$

Proof. The linear map taking a polynomial to its unique normal form by the division algorithm on $\mathcal{G}_{\prec}(I)$ induces an isomorphism of $k$-vector spaces

$$
R_{a} / I_{a} \rightarrow R_{a} / \operatorname{in}_{\prec}(I)_{a} .
$$

Consider a shared facet of the cones $C_{1}$ and $C_{2}$ in the Gröbner fan with a relative interior point $v$. The "edge ideal" $\operatorname{in}_{v}(I)$ is homogeneous with respect to any vector in the relative interior of the facet and consequently also homogeneous with respect to any vector in the span of the facet. Since $C_{1}$ and $C_{2}$ both contain positive vectors, so does $\operatorname{span}_{\mathbb{R}}\left(C_{v}(I)\right)$. Recall that $C_{v}(I)$ is the closure of the equivalence class of $v$. Pick a basis $u_{1}, \ldots, u_{n-1} \in \mathbb{N}^{n}$ for $\operatorname{span}_{\mathbb{R}}\left(C_{v}(I)\right)$ with $u_{1}$ being a positive vector. The vectors induce a grading $\mathcal{A}_{v}: \mathbb{N}^{n} \rightarrow \mathbb{N}^{n-1}$ on $R$ by

$$
\mathcal{A}_{v}(b)=\left(\left\langle u_{1}, b\right\rangle, \ldots,\left\langle u_{n-1}, b\right\rangle\right)
$$

for $b \in \mathbb{N}^{n}$. The initial ideal $\operatorname{in}_{v}(I)$ is $\mathcal{A}_{v}$-homogeneous.

Lemma 3.10. Let $\prec$ be a term order, $I$ an ideal, $\left(C_{1}, C_{2}\right)$ a directed edge with respect to the orientation in Definition 3.4 and $M_{1}$ and $M_{2}$ the initial ideals of $C_{1}$ and $C_{2}$ respectively. Let $v$ be a relative interior point in the shared facet. Then $\operatorname{in}_{\prec}\left(\operatorname{in}_{v}(I)\right)=M_{2}$.

Proof. Choose a positive interior point $\omega_{2}$ of $C_{2}$. We claim that the following identities hold:

$$
M_{2}=\operatorname{in}_{\omega_{2}}(I)=\operatorname{in}_{\prec_{\omega_{2}}}\left(\operatorname{in}_{\omega_{2}}(I)\right)=\operatorname{in}_{\prec_{\omega_{2}}}(I)=\operatorname{in}_{\prec_{\omega_{2}}}\left(\operatorname{in}_{v}(I)\right)=\operatorname{in}_{\prec}\left(\operatorname{in}_{v}(I)\right) .
$$

The first one holds by the choice of $\omega_{2}$. The second one is clear since $\operatorname{in}_{\omega_{2}}(I)$ is a monomial ideal. The third one holds by Lemma 2.13 and Lemma 2.15. By Lemma 2.13 the fourth equality holds since $v \in C_{\prec_{\omega_{2}}}(I)=C_{\omega_{2}}(I)$. To prove the last equality we look at the reduced Gröbner basis $\mathcal{G}_{\prec}\left(\operatorname{in}_{v}(I)\right)$. If we can show that $\operatorname{in}_{\prec_{\omega_{2}}}(g)=\operatorname{in}_{\prec}(g)$ for all elements $g \in \mathcal{G}_{\prec}\left(\operatorname{in}_{v}(I)\right)$, then we know that $\mathcal{G}_{\prec}\left(\operatorname{in}_{v}(I)\right)$ is also a Gröbner basis with respect to $\prec_{\omega_{2}}$ and the generators for the initial ideal 
$\operatorname{in}_{\prec_{\omega_{2}}}\left(\operatorname{in}_{v}(I)\right)$ are exactly the same as those for $\operatorname{in}_{\prec}\left(\operatorname{in}_{v}(I)\right)$. This would complete the proof.

The reduced Gröbner basis $\mathcal{G}_{\prec}\left(\operatorname{in}_{v}(I)\right)$ is $\mathcal{A}_{v}$-homogeneous. For an element $g$ this implies that the difference between two of its exponent vectors must be perpendicular to the shared facet. By Definition 3.4 there exists a normal $q-p$ of the facet with $\mathbf{x}^{p} \prec \mathbf{x}^{q}$ and $\left\langle\omega_{2}, q-p\right\rangle>0$. Since $\prec$ and $\prec \omega_{2}$ agree on one normal vector they must agree on all exponent differences of elements in $\mathcal{G}_{\prec}\left(\operatorname{in}_{v}(I)\right)$.

Notice that by Proposition 3.9 any initial ideal $\operatorname{in}_{\prec}\left(\operatorname{in}_{v}(I)\right)$ of $\operatorname{in}_{v}(I)$ has the same $\mathcal{A}_{v}$-graded Hilbert function as $\operatorname{in}_{v}(I)$.

By a flip we mean a move from one vertex in the graph to a neighbor. For a degree $a \in \mathbb{N}^{n-1}$ we call $\mathcal{A}_{v}^{-1}(a)$ the fiber over $a$. The $\mathcal{A}_{v}$-graded Hilbert function of an initial ideal $\operatorname{in}_{\prec}\left(\operatorname{in}_{v}(I)\right)$ counts the number of standard monomials inside each fiber. A flip preserves the Hilbert function. We may think of this as monomials in the monomial initial ideal moving around in the fiber. We wish to keep track of how the monomials move when we walk in the oriented graph. We define exactly what we mean by "moving around":

Definition 3.11. Let $\prec, M_{1}, M_{2}, u_{1}, \ldots, u_{n-1}$ and let $v$ be as above with

$$
\operatorname{in}_{\prec}\left(\operatorname{in}_{v}(I)\right)=M_{2} .
$$

Let $N_{1}$ and $N_{2}$ be the monomials in $M_{1}$ and $M_{2}$ respectively. We define the bijection $\phi_{\prec M_{1} M_{2}}: N_{1} \rightarrow N_{2}$ in the following way: For a monomial $\mathbf{x}^{b} \in N_{1}$ look at the monomials $B_{1} \subseteq N_{1}$ and $B_{2} \subseteq N_{2}$ with the same $\mathcal{A}$-degree as $\mathbf{x}^{b}$. Since taking initial ideals preserves the $\mathcal{A}$-graded Hilbert function, $\left|B_{1}\right|=\left|B_{2}\right|$. Sort $B_{1}$ and $B_{2}$ with respect to $\prec$. The bijection $\phi_{\prec M_{1} M_{2}}$ is now defined by taking the first element of $B_{1}$ to the first element of $B_{2}$, the second element of $B_{1}$ to the second element of $B_{2}$ and so on.

The following lemma is from [17, Lemma 4.1]:

Lemma 3.12. Let $\leq_{1}$ and $\leq_{2}$ be two term orders. If $f_{1}^{1}, \ldots, f_{s}^{1}$ is a vector space basis for $I_{a}$ such that $\mathrm{in}_{\leq_{1}}\left(f_{1}^{1}\right), \ldots, \mathrm{in}_{\leq_{1}}\left(f_{s}^{1}\right)$ is a basis for $\mathrm{in}_{\leq_{1}}(I)_{a}$, then there exists a basis $f_{1}^{2}, \ldots, f_{s}^{2}$ for $I_{a}$ such that $\operatorname{in}_{\leq_{2}}\left(f_{1}^{2}\right), \ldots, \mathrm{in}_{\leq_{2}}\left(f_{s}^{2}\right)$ is a basis for $\operatorname{in}_{\leq_{2}}(I)_{a}$ and

$$
\begin{gathered}
\operatorname{in}_{\leq_{2}}\left(f_{1}^{2}\right) \leq_{1} \operatorname{in}_{\leq_{1}}\left(f_{1}^{1}\right) \\
\vdots \\
\operatorname{in}_{\leq_{2}}\left(f_{s}^{2}\right) \leq_{1} \operatorname{in}_{\leq_{1}}\left(f_{s}^{1}\right) .
\end{gathered}
$$

Corollary 3.13. Let the setting be as in Definition 3.11. Then if $\mathbf{x}^{b} \in M_{1}$, we have $\phi_{\prec M_{1} M_{2}}\left(\mathbf{x}^{b}\right) \nprec \mathbf{x}^{b}$.

Proof. Let $a$ be the $\mathcal{A}$-degree of $\mathbf{x}^{b}$. We apply Lemma 3.12 with $I$ in the lemma being $\operatorname{in}_{v}(I)$. Let $\leq_{1}$ be $\prec$ and $\leq_{2}$ be the refinement of the preorder induced by $u_{1}$ with the reversed order of $\prec$. By the orientation of the graph, $M_{1}=\operatorname{in}_{\leq_{2}}\left(\operatorname{in}_{v}(I)\right)$ and $M_{2}=\operatorname{in}_{\leq_{1}}\left(\operatorname{in}_{v}(I)\right)$. By multiplying elements of $\mathcal{G}_{\prec}\left(\operatorname{in}_{v}(I)\right)$ by monomials we can construct a $k$-basis $f_{1}^{1}, \ldots, f_{s}^{1}$ of $\operatorname{in}_{v}(I)_{a}$ with $\operatorname{in}_{\leq_{1}}\left(f_{1}^{1}\right), \ldots$, in $_{\leq_{1}}\left(f_{s}^{1}\right)$ being a basis of $\left(M_{2}\right)_{a}$. By the lemma there is a basis $\operatorname{in}_{\leq_{2}}\left(f_{1}^{2}\right), \ldots$, in $_{\leq_{2}}\left(f_{s}^{2}\right)$ of $\left(M_{1}\right)_{a}$. Sort the list of inequalities in the lemma with $\operatorname{in}_{\leq_{2}}\left(f_{i}^{2}\right)$ decreasing w.r.t. $\prec\left(\leq_{1}\right)$. The right-hand side can now be sorted with respect to the same order without 
violating the inequalities. To see this, use the bubble sort algorithm: when two adjacent inequalities are swapped ...

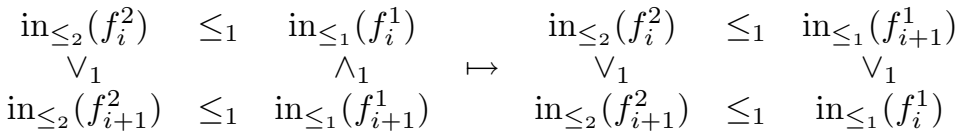

... the relations on the right-hand side of the arrow hold by transitivity of $\leq_{1}$.

After sorting, $\mathbf{x}^{b}$ appears somewhere on the left and $\phi_{\prec M_{1} M_{2}}\left(\mathbf{x}^{b}\right)$ on the right in the same inequality. This completes the proof.

Proof of Theorem 3.6. Suppose $C_{1}, C_{2}, \ldots, C_{m}$ was a path in the oriented graph with $C_{1}=C_{m}$. Let $M_{1}, \ldots, M_{m}$ denote the initial ideals and $N_{1}, \ldots, N_{m}$ their monomials. We will prove that the bijection $\phi:=\phi_{\prec M_{m-1} M_{m}} \circ \cdots \circ \phi_{\prec M_{1} M_{2}}$ is the identity on $M_{1}$. Suppose it is not the identity and let $\mathbf{x}^{b}$ be the smallest element in $M_{1}$ with respect to $\prec$ that is not fixed by $\phi$. By Corollary 3.13, $\mathrm{x}^{b}$ is the image of a smaller element in $M_{1}$ with respect to $\prec$. But this element is fixed by the minimality of $\mathbf{x}^{b}-$ a contradiction. The composition being the identity implies by Corollary 3.13 that $\phi_{\prec M_{i} M_{i+1}}$ is the identity for all $i$. Hence $M_{i}=M_{i+1}$, contradicting that $M_{1}, M_{2}, \ldots, M_{m}$ is a path.

We claim that $C_{\prec}(I)$ is the unique sink. If $v$ is in the relative interior of a facet of $C_{\prec}(I)$, then by Lemma $2.13 \operatorname{in}_{\prec}\left(\operatorname{in}_{v}(I)\right)=\operatorname{in}_{\prec}(I)$. By Lemma 3.10 this means that all edges connected to $C_{\prec}(I)$ are ingoing. Hence $C_{\prec}(I)$ is a sink.

To prove uniqueness let $C_{\prec^{\prime}}(I)$ be some sink in the oriented graph. By [19] has a matrix representation $\left(\tau_{0}, \ldots, \tau_{n-1}\right) \in \mathbb{R}^{n \times n}$ such that $\tau_{\varepsilon}:=\tau_{0}+\varepsilon \tau_{1}+\cdots+$ $\varepsilon^{n-1} \tau_{n-1} \in \operatorname{int} C_{\prec}(I)$ for $\varepsilon>0$ sufficiently small. Furthermore, for any $f \in R$, $\operatorname{in}_{\tau_{\varepsilon}}(f)=\operatorname{in}_{\prec}(f)$ for $\varepsilon>0$ sufficiently small. If $C_{\prec^{\prime}}(I)$ is a sink, then according to Definition 3.4 there exists a complete list of inner normals $q_{1}-p_{1}, \ldots, q_{r}-p_{r}$ of $C_{\prec^{\prime}}(I) \cap \mathbb{R}_{\geq 0}^{n}$ such that $\operatorname{in}_{\prec}\left(\mathbf{x}^{q_{i}}-\mathbf{x}^{p_{i}}\right)=\mathbf{x}^{q_{i}}$. Since $\tau_{\varepsilon}$ and $\prec$ pick out the same initial forms on a finite set of polynomials for $\varepsilon>0$ sufficiently small we see that $\left\langle\tau_{\varepsilon}, q_{i}\right\rangle>\left\langle\tau_{\varepsilon}, p_{i}\right\rangle$ or, equivalently, $\tau_{\varepsilon} \in \operatorname{int} C_{\prec^{\prime}}(I)$ for $\varepsilon>0$ sufficiently small. We conclude that $C_{\prec^{\prime}}(I)=C_{\prec}(I)$.

\section{IMPLEMENTATION ISSUES}

We can find a single Gröbner cone by applying Buchberger's algorithm and Corollary 2.11 for some term order. Since the graph of the Gröbner fan of $I$ is connected we may choose any graph traversal algorithm for computing the fulldimensional Gröbner cones. To do the local computations we need to be able to find the edges (connecting facets) of a full-dimensional cone and we need to be able to find the neighbor along an edge. We will see how to do this in the following sections.

Throughout the graph enumeration process we will represent the Gröbner cones by their marked reduced Gröbner bases, rather than by their defining inequalities, their term orders, etc. This choice is justified by the following known theorem, which we shall not prove:

Theorem 4.1. Let $I \subseteq R=k\left[x_{1}, \ldots, x_{n}\right]$ be an ideal. The marked reduced Gröbner bases of $I$, the monomial initial ideals of $I$ (w.r.t. a positive vector) and the fulldimensional Gröbner cones are in bijection. 
An important issue when implementing the algorithms is to identify shared facets. We say that a facet is flippable if its relative interior contains a positive vector. The flippable facets in a Gröbner fan are always shared. With the right definition of search edges the search tree will only consist of flippable facets.

At the end of the section we will see how the search edge computation in the reverse search algorithm can be implemented and we will explain how to take advantage of symmetry in a Gröbner fan traversal.

4.1. Finding facets. Suppose that we know a marked reduced Gröbner basis $\mathcal{G}_{\prec}(I)$ with respect to some unknown term order $\prec$. Proposition 2.6 (or Corollary 2.11) tells us how to read off the defining inequality system for $C_{\prec}(I)$.

Since $C_{\prec}(I)$ is full-dimensional the system contains no equations but only inequalities. Some of these inequalities are equivalent in the sense that they are multiples of each other. Taking just one inequality from each equivalence class the problem is now to find irredundant facet normals of a cone - or equivalently to find the extreme rays of the dual cone. Checking if a ray is extreme can be done by linear programming.

Not all of the remaining inequalities are guaranteed to define flippable facets. One way to ensure that we only get flippable facets is by adding the constraints $e_{i} \cdot x \geq 0$ for $i=1, \ldots, n$ and ignoring the facets defined by these.

A more efficient method (on some examples) is to find all facets and then remove the non-flippable irredundant facet normals by explicit checks. In our implementation this is done by checking if the inequality system with the inequality in question inverted still has a positive solution.

As mentioned in 13 there is an algebraic test that helps us eliminate redundant inequalities of $C_{\prec}(I)$. Let $\alpha \in \mathbb{R}^{n}$ be a coefficient vector of an inequality. If $\alpha$ is indeed irredundant and defines a facet with a relative interior point $v$, then Corollary 2.14 tells us how to compute $\mathcal{G}_{\prec}\left(\operatorname{in}_{v}(I)\right)$. This marked reduced Gröbner basis can be computed from $\mathcal{G}_{\prec}(I)$ as $\left\{\operatorname{in}_{v}(g)\right\}_{g \in \mathcal{G}_{\prec}(I)}$ if we just know $\alpha$ and not necessarily $v$; see the next section. A necessary condition for $\alpha$ to be irredundant is that the computed set $\left\{\operatorname{in}_{v}(g)\right\}_{g \in \mathcal{G}_{\prec}(I)}$ indeed is a marked Gröbner basis; i.e., all S-polynomials reduce to zero. This check even works for $v$ outside the positive orthant. A quicker necessary condition that we can check is that every non-zero S-polynomial should have at least one of its terms in in $\prec(I)$. For huge sets of inequalities the test works extremely well: 500 inequalities might reduce to 50 of which maybe 10 are irredundant. Our experience is that having this test as a preprocessing step can be much faster than solving the full linear programs with exact arithmetic.

4.2. Local change. Let $\mathcal{G}_{\prec}(I)$ be a known marked Gröbner basis and let $F$ be a flippable facet of $C_{\prec}(I)$. We let flip $\left(\mathcal{G}_{\prec}(I), F\right)$ denote the unique reduced Gröbner basis different from $\mathcal{G}_{\prec}(I)$ whose Gröbner cone also has $F$ as a facet. We will describe an algorithm for computing flip $\left(\mathcal{G}_{\prec}(I), F\right)$ given $\mathcal{G}_{\prec}(I)$ and an inner normal vector $\alpha$ for $F$. For a marked Gröbner basis $\mathcal{G}$ and a polynomial $g$ we let $g^{\mathcal{G}}$ denote the normal form of $g$ modulo $\mathcal{G}$ and note that this form does not depend on the term order but only on $\mathcal{G}$.

\section{Algorithm 4.2.}

Input: $A$ marked reduced Gröbner basis $\mathcal{G}_{\prec}(I)$ with $\prec$ being an unknown term order and an inner normal vector $\alpha$ of a flippable facet $F$ of $C_{\prec}(I)$. 
Output: $\mathcal{G}=\operatorname{flip}\left(\mathcal{G}_{\prec}(I), F\right)$.

\{

Let $v$ be a positive vector in the relative interior of $F$;

Compute $\mathcal{G}_{\prec}\left(\operatorname{in}_{v}(I)\right)=\left\{\operatorname{in}_{v}(g): g \in \mathcal{G}_{\prec}(I)\right\}$;

Compute the marked basis $\mathcal{G}_{\prec-\alpha}\left(\operatorname{in}_{v}(I)\right)$ from $\mathcal{G}_{\prec}\left(\operatorname{in}_{v}(I)\right)$ using Buchberger's algorithm;

$\mathcal{G}:=\left\{g-g^{\mathcal{G} \prec(I)}: g \in \mathcal{G}_{\prec-\alpha}\left(\operatorname{in}_{v}(I)\right)\right\} ;$

Mark the term in $_{{ }_{-\alpha}}(g)$ in each element $g-g^{\mathcal{G} \prec(I)}$ in $\mathcal{G}$;

Turn $\mathcal{G}$ into a reduced basis;

\}

The algorithm is a special case of the local change procedure for a single step in the Gröbner walk [6. See [10, Proposition 3.2] for a new treatment and a proof. Here we will just add a few comments on our special case - the case where $F$ is a facet and not a lower-dimensional face.

For any vector $\omega$ in the relative interior of $F, \operatorname{in}_{\omega}(I)=\operatorname{in}_{v}(I)$ is homogeneous with respect to the $\omega$-grading. Since $F$ is $(n-1)$-dimensional, $\operatorname{in}_{v}(I)$ is homogeneous with respect to all vectors inside $\operatorname{span}_{\mathbb{R}}(\alpha)^{\perp}$. All Gröbner bases of $\operatorname{in}_{v}(I)$ are homogeneous in the same way. Consequently, each of them must consist of polynomials of the form $\sum_{s=0}^{t} c_{s} \mathbf{x}^{(a+s b)}$, where $a \in \mathbb{N}^{n}$ and $b \in \mathbb{Z}^{n}$ is parallel to $\alpha$. The same is true for all polynomials appearing in any run of Buchberger's algorithm starting from one of these sets. A consequence is that in order to run Buchberger's algorithm we only need to decide if we are in the situation where $\mathbf{x}^{\gamma} \prec \mathbf{x}^{\gamma+\alpha}$ for $\gamma \in \mathbb{N}^{n}$ or in the situation where $\mathbf{x}^{\gamma+\alpha} \prec \mathbf{x}^{\gamma}$ for $\gamma \in \mathbb{N}^{n}$. Thus specifying $\alpha$ or $-\alpha$ as a term order suffices - no tie-breaker is needed. The initial ideal $\operatorname{in}_{v}(I)$ can have at most two reduced Gröbner bases. Both term orders are legal since $\operatorname{in}_{v}(I)$ is homogeneous with respect to the strictly positive vector $v$.

The Gröbner basis $\mathcal{G}_{\prec}\left(\mathrm{in}_{v}(I)\right)$ can be read off from the marked Gröbner basis $\mathcal{G}_{\prec}(I)$ by taking initial forms of the polynomials with respect to $v$; see Corollary 2.14 Taking the initial form $\operatorname{in}_{v}(g)$ of a polynomial $g \in \mathcal{G}_{\prec}(I)$ without computing $v$ is done as follows. By Corollary 2.11, in $\operatorname{in}_{\prec}\left(\operatorname{in}_{v}(g)\right)=\operatorname{in}_{\prec}(g)$ and thus we already know one term of $\operatorname{in}_{v}(g)$ since $\operatorname{in}_{\prec}(g)$ is the marked term of $g$ in $\mathcal{G}_{\prec}(I)$. Since every $\omega$ in the relative interior of $F$ will have $\mathcal{G}_{\prec}\left(\operatorname{in}_{\omega}(I)\right)=\mathcal{G}_{\prec}\left(\operatorname{in}_{v}(I)\right)$ the remaining terms of $\operatorname{in}_{v}(g)$ are exactly the terms in $g$ with the same $\omega$-degree as $\operatorname{in}_{\prec}(g)$ for all $\omega$ in the relative interior of $F$ and consequently for all $\omega$ in $\operatorname{span}_{\mathbb{R}}(\alpha)^{\perp}$. In other words, a term of $g$ is in $\operatorname{in}_{v}(g)$ if and only if its exponent vector minus the exponent of $\operatorname{in}_{\prec}(g)$ is parallel to $\alpha$. The term order $\prec$ does not have to be known for this step, nor does it have to be known in the computation of $\mathcal{G}_{\prec-\alpha}\left(\operatorname{in}_{v}(I)\right)$ or in any other subsequent step. The vector $v$ also remains unknown in the entire process.

4.3. Computing the search edge. Let $\prec$ be the term order used for orienting the graph of the Gröbner fan. In Algorithm 3.2 the search edge $e_{C_{\prec^{\prime}}(I)}$ has to be computed given $\mathcal{G}_{\prec^{\prime}}(I)$, where $\prec^{\prime}$ is some unspecified term order. According to Proposition 3.1 the definition of search edges can be arbitrary. However, efficiently computing a search edge requires a good definition. Our search edges will always come from flippable facets.

One strategy for locally computing the search edge $e_{C_{\prec^{\prime}}(I)}$ is to compute a unique representation of each flippable facet of the Gröbner cone $C_{\prec^{\prime}}(I)$ and then choose 
the smallest of these facets to be $e_{\mathcal{C}_{\alpha^{\prime}}(I)}$ in some lexicographic order. This method requires all facets to be computed every time we check if " $e_{u}=(u, v)$ " in Algorithm 3.2 .

A better strategy is to draw a straight line from a point in the cone $C_{\prec^{\prime}}(I)$ to the cone of the sink and choose the first facet intersecting this line as $e_{C_{\prec^{\prime}}(I)}$. A point in the cone $C_{\prec^{\prime}}(I)$ can be computed deterministically by linear programming. Two problems arise. The straight line might not intersect a unique facet, and we may not know a point in the cone of the sink. Both problems can be solved using formal perturbation of the end points of the line. This was worked out in detail in [10. Here we explain how it works for lexicographic term orders and with one endpoint perturbed.

Lemma 4.3. Let $I \subseteq R$ be an ideal and $\prec$ the lexicographic term order with $x_{1} \succ x_{2} \succ \cdots \succ x_{n}$. Define $\tau_{\varepsilon}=\left(\varepsilon^{0}, \varepsilon^{1}, \ldots, \varepsilon^{n-1}\right)$. There exists a $\delta>0$ such that $\operatorname{in}_{\tau_{\varepsilon}}(I)=\operatorname{in}_{\prec}(I)$ for all $\varepsilon \in(0, \delta)$.

Proof. This follows from Lemma 2.10 since $\prec$ and $\tau_{\varepsilon}$ agree on a finite set of polynomials for small $\varepsilon$.

Let $\sigma$ be a deterministically computed interior point of the cone of $C_{\prec^{\prime}}(I)$ and assume for simplicity that $\sigma \in \mathbb{N}^{n}$. For sufficiently small $\varepsilon>0$ the line segment

$$
\omega(t):=(1-t) \sigma+t \tau_{\varepsilon} \text { with } t \in[0,1]
$$

intersects a facet of $C_{\prec^{\prime}}(I)$ unless $C_{\prec^{\prime}}(I)$ is the sink.

Let $\left\{\alpha_{1}, \ldots, \alpha_{m}\right\}$ be the set of potential inner facet normals read off from $\mathcal{G}_{\prec^{\prime}}(I)$. We are only interested in the vectors $\alpha_{i}$, where $\left\langle\sigma, \alpha_{i}\right\rangle>0$ and $\left\langle\tau_{\varepsilon}, \alpha_{i}\right\rangle<0$. Let $t_{i}$ denote the $t$-value for the intersection of the line segment and the hyperplane defined by $\alpha_{i}$. Then

$$
t_{i}:=\frac{\left\langle\sigma, \alpha_{i}\right\rangle}{\left\langle\sigma, \alpha_{i}\right\rangle-\left\langle\tau_{\varepsilon}, \alpha_{i}\right\rangle}
$$

We wish to find $i$ such that $t_{i}$ is smallest (for small $\varepsilon$ ). We have

$$
\begin{aligned}
& t_{i}<t_{j} \Longleftrightarrow \\
& \frac{\left\langle\sigma, \alpha_{i}\right\rangle}{\left\langle\sigma, \alpha_{i}\right\rangle-\left\langle\tau_{\varepsilon}, \alpha_{i}\right\rangle}<\frac{\left\langle\sigma, \alpha_{j}\right\rangle}{\left\langle\sigma, \alpha_{j}\right\rangle-\left\langle\tau_{\varepsilon}, \alpha_{j}\right\rangle} \Longleftrightarrow \\
& \frac{\left\langle\sigma, \alpha_{i}\right\rangle-\left\langle\tau_{\varepsilon}, \alpha_{i}\right\rangle}{\left\langle\sigma, \alpha_{i}\right\rangle}>\frac{\left\langle\sigma, \alpha_{j}\right\rangle-\left\langle\tau_{\varepsilon}, \alpha_{j}\right\rangle}{\left\langle\sigma, \alpha_{j}\right\rangle} \Longleftrightarrow \\
& \frac{\left\langle\tau_{\varepsilon}, \alpha_{i}\right\rangle}{\left\langle\sigma, \alpha_{i}\right\rangle}<\frac{\left\langle\tau_{\varepsilon}, \alpha_{j}\right\rangle}{\left\langle\sigma, \alpha_{j}\right\rangle} \Longleftrightarrow \\
& \left\langle\tau_{\varepsilon},\left\langle\sigma, \alpha_{j}\right\rangle \alpha_{i}\right\rangle<\left\langle\tau_{\varepsilon},\left\langle\sigma, \alpha_{i}\right\rangle \alpha_{j}\right\rangle \Longleftrightarrow \\
& \mathbf{x}^{\left\langle\sigma, \alpha_{j}\right\rangle \alpha_{i}} \prec \mathbf{x}^{\left\langle\sigma, \alpha_{i}\right\rangle \alpha_{j}} .
\end{aligned}
$$

We see that for $\varepsilon$ sufficiently small " $t_{i}<t_{j}$ " does not depend on $\varepsilon$. Furthermore, there cannot be any ties, unless $\alpha_{i}$ and $\alpha_{j}$ represent the same hyperplane. This gives an easy method for defining and computing $e_{C_{\prec^{\prime}}(I)}$. We simply choose the facet defined by $a_{i}$ where $t_{i}$ is smallest among $\left\{t_{1}, \ldots, t_{m}\right\}$ (for small $\varepsilon>0$ ). 
4.4. Exploiting symmetry. In this section we explain how to take advantage of symmetry to speed up computations. The symmetric group $S_{n}$ acts on polynomials and ideals of $R$ by permuting variables and on $\mathbb{R}^{n}$ by permuting coordinate entries. Let $I \subseteq R$ be an ideal. We call a subgroup $\Gamma \leq S_{n}$ a symmetry group for $I$ if $\pi(I)=I$ for all $\pi \in \Gamma$. If we know a symmetry group for $I$ we can enumerate the reduced Gröbner bases of $I$ up to symmetry. Let $\Gamma$ be such a symmetry group for $I$.

In our description all Gröbner bases will be marked and reduced. Thereby each one will uniquely represent its initial ideal and Gröbner cone. For a Gröbner basis $\mathcal{G}$ of $I$ we use the notation $\Gamma_{\mathcal{G}}=\{\pi(\mathcal{G})\}_{\pi \in \Gamma}$ for its orbit.

The idea is to exploit the identity $\operatorname{flip}(\pi(\mathcal{G}), \pi(F))=\pi(\operatorname{flip}(\mathcal{G}, F))$ for all $\pi \in \Gamma$. In other words, $\Gamma$ is a group of automorphisms of the graph of the Gröbner fan of $I$. The quotient graph is defined to be the graph whose vertices are the orbits of Gröbner bases with two orbits $\Gamma_{\mathcal{G}}$ and $\Gamma_{\mathcal{G}^{\prime}}$ being connected if there exists a facet $F$ of the Gröbner cone of $\mathcal{G}$ such that $\operatorname{flip}(\mathcal{G}, F) \in \Gamma_{\mathcal{G}^{\prime}}$. The flip graph may have loops.

The symmetry-exploiting algorithm enumerates the quotient graph by a breadthfirst traversal. Orbits are represented by Gröbner basis representatives. One question that arises is how to check if two Gröbner bases $\mathcal{G}$ and $\mathcal{G}^{\prime}$ represent the same orbit. A solution is to run through all elements $\pi \in \Gamma$ and check if $\pi(\mathcal{G})$ equals $\mathcal{G}^{\prime}$, or even better to make a similar check for the monomial initial ideals. Although this does not seem efficient, it is still much faster in practice than redoing symmetric Gröbner basis and polyhedral computation as we have done in the usual reverse search or breadth-first enumeration without symmetry. It is not clear how to combine symmetry-exploiting with reverse search.

\section{Complexity}

In this section we will discuss the complexity of enumerating the maximal cones of the Gröbner fan of an ideal $I$ by reverse search. We will assume that $I$ is homogeneous with respect to a positive vector. This guarantees that any facet of a full-dimensional Gröbner cone is flippable.

We identify the following important sub-algorithms:

- Computation of the facet normals of the Gröbner cone of a marked reduced Gröbner basis $\mathcal{G}$. We denote the time for this operation by $T_{\text {facets }}(\mathcal{G})$.

- Computation of a search edge given a marked reduced Gröbner basis $\mathcal{G}$ as described in Subsection 4.3. We denote the time for this operation by $T_{\text {shoot }}(\mathcal{G})$.

- Conversion of a marked reduced Gröbner basis $\mathcal{G}_{1}$ into a marked reduced Gröbner basis $\mathcal{G}_{2}$, where the Gröbner cones of $\mathcal{G}_{1}$ and $\mathcal{G}_{2}$ are assumed to share a facet. We denote the time for this operation by $T_{\text {flip }}\left(\mathcal{G}_{1}, \mathcal{G}_{2}\right)$.

For simplicity we will assume that the time used for solving any of the linear programs in our algorithms only depends on the dimensions of its matrix form. We let $T_{\mathbf{l}}(n, r)$ be the time needed to solve a linear programming problem with $n$ variables and $r$ constraints. Then $T_{\text {facets }}(\mathcal{G})$ and $T_{\text {shoot }}(\mathcal{G})$ can be expressed in terms of $T_{\mathbf{l}}(n, r)$.

The time $T_{\text {facets }}(\mathcal{G}) \in O\left(T_{\mathbf{l}}(n, r) r\right)$, where $r$ is the number of non-leading terms in $\mathcal{G}$. The reason is that each non-leading term in $\mathcal{G}$ gives an inequality in the description of the Gröbner cone. Checking if the inequality defines a facet takes 
one linear program. In addition, duplicates should be removed from the set of facet normals and further vectors should be eliminated until no parallel vectors exist. The time for this step is dominated by the time for solving LPs.

The time $T_{\text {shoot }}(\mathcal{G}) \in O\left(r n^{2}+T_{\mathbf{l p}}(n, r)\right)$, where $r$ is the number of non-leading terms in $\mathcal{G}$. The first step in the algorithm is to deterministically find a relative interior point of the Gröbner cone. This is done in time $T_{\mathbf{l}}(n, r)$. After this the smallest vector among the $r$ defining vectors for the cone with respect to the ordering in Subsection 4.3 needs to be found. Comparing two vectors takes $O\left(n^{2}\right)$ operations in the worst case. These are operations in $\mathbb{Q}$. In the above estimate we assume that each operation takes constant time.

We have no good bound for the complexity of flipping. Now we count the number of times each of the three sub-algorithms is applied when enumerating the graph of the Gröbner fan of $I$ using reverse search.

- The facets of each Gröbner cone are computed exactly once in Algorithm 3.2 (right after the Gröbner basis has been output). We remark that since we are only interested in facets with the correct orientation the number of LPs that really need to be solved is lower than the $r$ in the discussion above. We will not take this into account in our analysis.

- Checking if an edge is a search edge is done once for every edge. Each time we need to recompute a search edge and compare it to the edge. Hence the total number of times we need to compute a search edge is equal to the number of edges in the graph of the Gröbner fan of $I$.

- When a vertex $\mathcal{G}$ is processed by Algorithm 3.2 we must test for every ingoing edge if the edge is a search edge. To test this we first compute flip $(\mathcal{G}, F)$, where $F$ is the facet of the cone corresponding to the edge in question. If the edge is equal to the search edge of flip $(\mathcal{G}, F)$, we do an enumeration of the subtree with root $\operatorname{flip}(\mathcal{G}, F)$. If not, $\operatorname{flip}(\mathcal{G}, F)$ is forgotten. Since all vertices are processed once and every edge is ingoing for exactly one vertex the number of times flip $(\mathcal{G}, F)$ needs to be computed is equal to the number of edges in the graph. We remark that the variant of the reverse search where the search path for the current vertex is not stored on the recursion stack would require twice as many computations of this kind.

Let $E$ be the edges and $V$ be the vertices of the graph. The total time complexity of the enumeration of $(V, E)$ is:

$$
O\left(\sum_{\mathcal{G} \in V} T_{\text {facets }}(\mathcal{G})+\sum_{\left(\mathcal{G}_{1}, \mathcal{G}_{2}\right) \in E} T_{\text {shoot }}\left(\mathcal{G}_{1}\right)+\sum_{\left(\mathcal{G}_{1}, \mathcal{G}_{2}\right) \in E} T_{\text {flip }}\left(\mathcal{G}_{1}, \mathcal{G}_{2}\right)\right) .
$$

Substituting with the time needed for solving the LPs we get the following theorem:

Theorem 5.1. Let $(V, E)$ be the graph of the Gröbner fan of I. The time complexity for computing this graph given a marked reduced Gröbner basis is in the class of functions

$$
O\left(\sum_{\mathcal{G} \in V} T_{\mathbf{l}}(n, r(\mathcal{G})) r(\mathcal{G})+\sum_{\left(\mathcal{G}_{1}, \mathcal{G}_{2}\right) \in E} r\left(\mathcal{G}_{1}\right) n^{2}+T_{\mathbf{l}}\left(n, \mathcal{G}_{1}\right)+\sum_{\left(\mathcal{G}_{2}, \mathcal{G}_{1}\right) \in E} T_{\text {flip }}\left(\mathcal{G}_{1}, \mathcal{G}_{2}\right)\right),
$$

where $r(\mathcal{G})$ is the number non-leading terms in the marked reduced Gröbner basis $\mathcal{G}$. In particular, the first two terms are bounded by a polynomial in the size of the output. 
Remark 5.2. If for a given class of ideals the time $T_{\text {flip }}\left(\mathcal{G}_{1}, \mathcal{G}_{2}\right)$ is bounded by a polynomial in the size of the binary encoding of $\mathcal{G}_{1}$ and $\mathcal{G}_{2}$, then the enumeration of the reduced Gröbner bases for an ideal in the class by reverse search is a polynomial time algorithm in the size of the output.

For a general polynomial ideal, the time $T_{\text {flip }}\left(\mathcal{G}_{1}, \mathcal{G}_{2}\right)$ is not expected to be bounded by a polynomial in the size of $\mathcal{G}_{1}$ and $\mathcal{G}_{2}$. Linear ideals and principal ideals are two trivial situations in which such a polynomial bound exists.

The process of converting a given Gröbner basis of an ideal to a different Gröbner basis of the same ideal is known in the literature as Gröbner basis conversion and has been studied in papers such as 6, 8 and 22. This process is the chief goal of the Gröbner walk 1, 6, 10. Algorithm 4.2 is a very special case of this conversion where we want to convert a given Gröbner basis to one of its neighbors in the Gröbner fan. In [16, Kalkbrener proves that for two adjacent Gröbner bases $\mathcal{G}_{1}$ and $\mathcal{G}_{2}$, the maximal degree of an element of $\mathcal{G}_{2}$ is bounded above by a quadratic polynomial in the maximal degree of the polynomials in $\mathcal{G}_{1}$. This does not a priori provide a polynomial bound for $T_{\text {flip }}\left(\mathcal{G}_{1}, \mathcal{G}_{2}\right)$.

\section{Computational Results and examples}

The algorithms presented in this paper were implemented in the software package Gfan [14]. In this section we present examples of Gröbner fans computed using this package. The first example comes with a picture and gives an idea of the kind of geometric shape a Gröbner fan might have.

Example 6.1. 21, Example 3.9] Consider the ideal $I=\left\langle a^{5}-1+c^{2}+b^{3}, b^{2}-1+\right.$ $\left.c+a^{2}, c^{3}-1+b^{5}+a^{6}\right\rangle \subseteq \mathbb{Q}[a, b, c]$. The Gröbner fan of $I$ has 360 full-dimensional cones and the Gröbner region is $\mathbb{R}_{\geq 0}^{3}$. The intersection of the fan with the standard simplex in $\mathbb{R}^{3}$ is shown in Figure 4 .

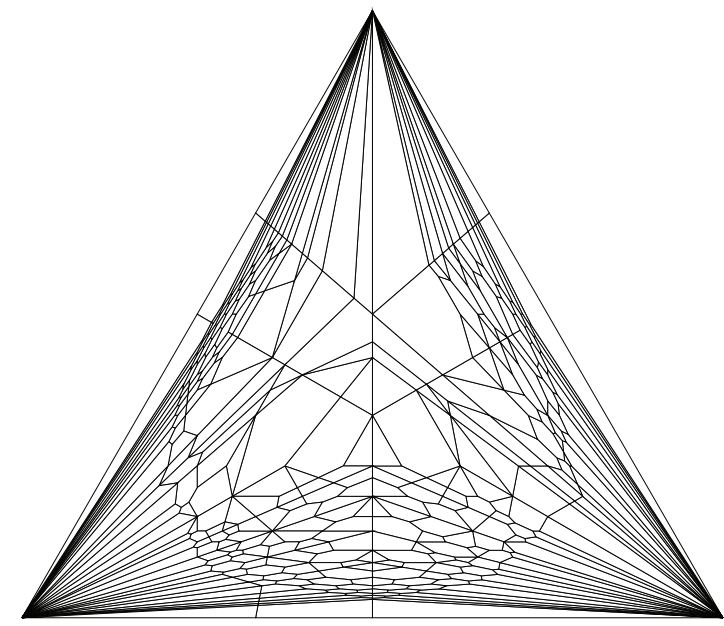

Figure 4. The Gröbner fan of the ideal in Example6.1 intersected with the standard 2 -simplex. The $a$-axis is on the right, the $b$-axis on the left and the $c$-axis at the top. 
TABLE 1. Statistics for the Gröbner fans computed using Gfan.

\begin{tabular}{|l|r|r|r|r|l|}
\hline Example & $n$ & $h$ & d & D & f-vector \\
\hline Det $_{3,3,4}$ & 12 & 6 & 3 & 3 & $(1,12,66,204,342,288,96)$ \\
Det $_{3,3,5}$ & 15 & 7 & 3 & 3 & $(1,45,585,3390,10710,19890,21750,12960,3240)$ \\
Det $_{3,4,4}$ & 16 & 7 & 3 & 5 & $(1, ?, ?, ?, ?, ?, ?, ?, ?, 163032)$ \\
Detsym $_{3,4}$ & 10 & 4 & 3 & 8 & $(1,518,5412,20505,36024,29808,9395)$ \\
Grass $_{2,5}$ & 10 & 5 & 2 & 3 & $(1,20,120,300,330,132)$ \\
Cyclic $_{5}$ & 5 & 0 & 8 & 15 & $(1, ?, ?, ?, ?, 55320)$ \\
J $_{4}$ & 4 & 1 & 3 & 8 & $(1,200,516,318)$ \\
\hline
\end{tabular}

We now list some families of ideals used in our computations. The Gröbner fans of these ideals have been computed for the parameters listed in Table 1 . The ambient field is always $\mathbb{Q}$. The columns of the table are to be interpreted as follows. In each row, the first column contains the name of the ideal (to be explained below). The second column lists $n$, the number of variables in the ideal. The third column lists $h$, the dimension of the lowest-dimensional Gröbner cone $C_{0}(I)$. Note that $h$ is the dimension of the homogeneity space of the ideal which is the common subspace contained in every Gröbner cone of the ideal. The quantity "d" is the lowest total degree of any reduced Gröbner basis of the ideal and "D" is the highest. The $f$ vector of the Gröbner fan is an ordered list of the number of $h$-dimensional cones, $(h+1)$-dimensional cones, etc., up to the number of $n$-dimensional cones.

Example 6.2. Let $\operatorname{Det}_{t, m, n}$ denote the ideal in the polynomial ring in $m n$ variables generated by the $t \times t$ minors of the matrix:

$$
\left(\begin{array}{cccc}
x_{11} & x_{12} & \cdots & x_{1 n} \\
x_{21} & x_{22} & \cdots & x_{2 n} \\
\vdots & \vdots & \ddots & \vdots \\
x_{m 1} & x_{m 2} & \cdots & x_{m n}
\end{array}\right) .
$$

Example 6.3. Let Grass $d, n$ denote the ideal in the polynomial ring in $\left(\begin{array}{l}n \\ d\end{array}\right)$ variables generated by the relations on the $d \times d$ minors of a $d \times n$ matrix.

Example 6.4. Let $\operatorname{Detsym}_{t, n}$ denote the ideal in the polynomial ring in $\frac{n(n+1)}{2}$ variables generated by the $t \times t$ minors of a symmetric matrix of variables. For example, Detsym ${ }_{3,4}$ is generated by the $3 \times 3$ minors of the following matrix:

$$
\left(\begin{array}{llll}
a & b & c & d \\
b & e & f & g \\
c & f & h & i \\
d & g & i & j
\end{array}\right) .
$$

Example 6.5. Let Cyclic $_{5}$ denote the ideal $\langle a+b+c+d+e, a b+b c+c d+d e+a e, a b c+$ $b c d+c d e+d e a+e a b, a b c d+a b c e+a b d e+a c d e+b c d e, a b c d e-1\rangle \subseteq k[a, b, c, d, e]$. In general, $\mathrm{Cyclic}_{n}$ stands for the generalization of this polynomial system to $n$ variables 4. These polynomial systems have become benchmarks for computer algebra packages and their lexicographic Gröbner bases are notoriously hard to compute. 
Example 6.6. Let $K_{n}$ denote the complete graph on $n$ vertices and $I_{n}$ be the Stanley-Reisner ideal of this graph. The Stanley-Reisner ideal of a simplicial complex $\Delta$ is the ideal generated by all monomials $x_{i_{1}} x_{i_{2}} \cdots x_{i_{n}}$ such that $\left\{i_{1}, \ldots, i_{n}\right\}$ is not a face of $\Delta$. Apply a generic linear change of coordinates to $I_{n}$ to obtain the ideal $J_{n}$. The generators of $J_{n}$ typically have very complicated coefficients. For example, the first generator in our $J_{4}$ was

$$
\begin{aligned}
& a^{3}+\frac{4980248985}{343338664} a^{2} c \quad+\frac{2079196217}{257503998} a b c \\
& -\frac{359584197}{171669332} b c^{2} \quad-\frac{84523033581}{1373354656} c^{3} \\
& +\frac{1194144014}{9656399925} b^{2} d+\frac{394500908221}{25750399800} a c d \\
& -\frac{4583330213}{3862559970} a d^{2}+\frac{181743499}{364392450} b d^{2} \\
& \begin{array}{rr}
+\frac{86858380}{128751999} b^{2} c & -\frac{2205648949}{42917333} a c^{2} \\
-\frac{11737327991}{51500799600} a^{2} d & -\frac{16299027451}{38625599700} a b d \\
-\frac{47953955497}{25750399800} b c d & +\frac{195491595943}{2985553600} c^{2} d \\
-\frac{429736138279}{25750399800} c d^{2} & +\frac{8566043731}{12875199900} d^{3} .
\end{array}
\end{aligned}
$$

The initial ideals of $J_{n}$ are known as the generic initial ideals of $I_{n}$. The reverse lexicographic generic initial ideals of an ideal have played an important role in commutative algebra and algebraic geometry while other generic initial ideals have not been explored very much. We computed the Gröbner fan of $J_{4}$.

Extracting the f-vector from the full-dimensional Gröbner cones produced in the enumeration process was the most time-consuming part of the computation of these examples. In example Det $_{3,3,4}$ this extraction was not possible to complete within a reasonable time with the current software package. For this particular example the 163032 full-dimensional Gröbner cones were computed up to the action of a symmetry group of order 576. The full-dimensional cones come in 289 orbits. The computation of the full-dimensional cones up to symmetry took 7 minutes on a $2.4 \mathrm{GHz}$ Pentium processor. Using reverse search without symmetry the same computation would take approximately 14 hours. The f-vector extraction routine in Gfan only works for complete fans. This is why the f-vector for the $\mathrm{Cyclic}_{5}$ example is not shown.

\section{REFERENCES}

1. Beatrice Amrhein, Oliver Gloor, and Wolfgang Küchlin, Walking faster, Proceedings DISCO '96 (Berlin), LNCS 1128, Springer, 1996, pp. 150-161.

2. David Avis and Komei Fukuda, Reverse search for enumeration, Discrete Applied Mathematics 65 (1996), 21-46. MR:1380066 (98a:05080)

3. David Bayer and Ian Morrison, Standard bases and geometric invariant theory I. Initial ideals and state polytopes., J. Symb. Comput. 6 (1988), no. 2/3, 209-217. MR.988413 (90e:13001)

4. Göran Björck and Ralf Fröberg, A faster way to count the solutions of inhomogeneous systems of algebraic equations, with applications to cyclic n-roots, J. Symb. Comput. 12 (1991), no. 3/4, 329-336. MR 1128248 (92g:68067)

5. Tristram Bogart, Anders Jensen, Rekha Thomas, David Speyer, and Bernd Sturmfels, Computing tropical varieties., J. Symb. Comput. 42 (2007), no. 1-2, 54-73. MR2284285

6. Stéphane Collart, Michael Kalkbrener, and Daniel Mall, Converting bases with the Gröbner walk., J. Symb. Comput. 24 (1997), no. 3/4, 465-469. MR1484492

7. David Cox, John Little, and Donal O'Shea, Ideals, varieties, and algorithms, second edition, Springer, 1997.

8. Jean-Charles Faugere, Patrizia Gianni, Daniel Lazard, and Teo Mora, Efficient computation of zero-dimensional Gröbner bases by change of ordering, J. Symb. Comput. 16 (1993), 329-344. MR:1263871 (94k:68095)

9. Komei Fukuda, cddlib reference manual, cddlib version 094b, Swiss Federal Institute of Technology, Lausanne and Zürich, Switzerland, 2005,

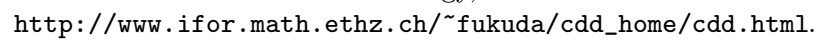

10. Komei Fukuda, Anders Jensen, Niels Lauritzen, and Rekha Thomas, The generic Gröbner walk., J. Symb. Comput. 42 (2007), no. 3, 298-312. 
11. William Fulton, Introduction to toric varieties, Princeton University Press, 1993. MR 1234037 (94g:14028)

12. Torbjörn Granlund et al., GNU multiple precision arithmetic library 4.1.2, December 2002, http://swox.com/gmp/.

13. Birkett Huber and Rekha R. Thomas, Computing Gröbner fans of toric ideals., Experimental Mathematics 9 (2000), no. 3/4, 321-331. MR1795304 (2001h:13039)

14. Anders N. Jensen, Gfan, a software system for Gröbner fans, Available at http://home.imf.au.dk/ajensen/software/gfan/gfan.html.

15. Anders N. Jensen, A non-regular Gröbner fan, Discrete and Computational Geometry 37 (2007), no. 3, 443-453.

16. Michael Kalkbrener, On the complexity of Gröbner bases conversion, J. Symb. Comput. 28 (1999), 265-273. MR.1709906 (2000g:13020)

17. Niels Lauritzen, Truncated Gröbner fans and lattice ideals,(2005).

18. Daniel Mall, Gröbner fans and projective schemes, Progress in Computer Science and Applied Logic 15 (1998), 181-191. MR1624580 (99d:13033)

19. Teo Mora and Lorenzo Robbiano, The Gröbner fan of an ideal., J. Symb. Comput. 6 (1988), no. 2/3, 183-208. MR988412 (90d:13004)

20. Jörg Rambau, TOPCOM: Triangulations of point configurations and oriented matroids, ZIB report 02-17 (2002).

21. Bernd Sturmfels, Gröbner bases and convex polytopes, University Lecture Series, vol. 8, American Mathematical Society, 1996. MR1363949 (97b:13034)

22. Carlo Traverso, Hilbert functions and the Buchberger algorithm, J. Symb. Comput. 22 (1996), 355-376. MR1428831 (98i:13054)

Institute for Operations Research and Institute of Theoretical Computer Science, ETH Zentrum, CH-8092 Zurich, Switzerland and Mathematics Institute / ROSO EPFL, CH-1015 Lausanne, Switzerland

E-mail address: fukuda@ifor.math.ethz.ch

Institut for Matematiske Fag, Aarhus Universitet, DK-8000 Århus, Denmark

E-mail address: ajensen@imf.au.dk

Department of Mathematics, University of Washington, Seattle, Washington 981954350

E-mail address: thomas@math. washington.edu 